\title{
Title: Linked Weyl Surfaces and Weyl Arcs in Photonic Metamaterials
}

Authors: Shaojie $\mathrm{Ma}^{1,3 \dagger}$, Yangang $\mathrm{Bi}^{1,3,4 \dagger}{ }^{4}$, Qinghua Guo ${ }^{5}$, Biao Yang ${ }^{6}$, Oubo You ${ }^{1,3}$, Jing Feng $^{4}$, Hong-Bo Sun ${ }^{4,7}$, Shuang Zhang ${ }^{1,2,3 *}$

\section{Affiliations:}

${ }^{1}$ Department of Physics, University of Hong Kong; Hong Kong, China.

${ }^{2}$ Department of Electrical \& Electronic Engineering, University of Hong Kong; Hong Kong, China.

${ }^{3}$ School of Physics and Astronomy, University of Birmingham; Birmingham B15 2TT, United Kingdom

${ }^{4}$ State Key Laboratory of Integrated Optoelectronics, College of Electronic Science and Engineering, Jilin University; 2699 Qianjin Street, Changchun 130012, China

${ }_{5}^{5}$ Department of Physics and Institute for Advanced Study, The Hong Kong University of Science and Technology; Hong Kong, China

${ }^{6}$ College of Advanced Interdisciplinary Studies, National University of Defense Technology; Changsha 410073, China

${ }^{7}$ State Key Laboratory of Precision Measurement Technology and Instruments, Department of Precision Instrument, Tsinghua University; Haidian District, Beijing 100084, China

*Corresponding author. Email: shuzhang@hku.hk

Abstract: Generalization of the concept of band topology from lower-dimensional to higherdimensional $(n>3)$ physical systems is expected to introduce new bulk and boundary topological effects. However, theoretically predicted topological singularities in five-dimensional systems - Weyl surfaces and Yang monopoles — have either not been demonstrated in realistic physical systems or are limited to purely synthetic dimensions. Here, we construct a system possessing Yang monopoles and Weyl surfaces based on metamaterials with engineered electromagnetic properties, leading to the observation of several intriguing bulk and surface phenomena, such as linking of Weyl surfaces and surface Weyl arcs, via selected threedimensional sub-spaces. The demonstrated photonic Weyl surfaces and Weyl arcs leverage the concept of higher-dimension topology to control the propagation of electromagnetic waves in artificially engineered photonic media.

One-Sentence Summary: An experimental demonstration of metamaterials exhibiting linked

Weyl surfaces and nontrivial boundary effects in 5D synthetic space. 


\section{Main Text:}

Gapless topological phases with various band crossings in crystals play an important role in topological physics as they host a suite of fascinating bulk and surface transport phenomena that include one-way propagation of energy and novel relativistic behaviors (1). As one of the most studied two-dimensional (2D) systems, graphene hosts Dirac points in the momentum space, which support massless quasi-particles and lie at the transition between different topological insulating phases $(2,3)$. The same band dispersion, when extended into three dimensions (3D), generalizes into Weyl points, which serve as the monopoles of Berry curvature - the momentum-space counterpart of magnetic field (4-8). Besides Weyl points, there exist other gapless topological phases in 3D systems, such as nodal lines and Dirac points. All the above 3D gapless phases greatly enrich the observed topology-related phenomena, such as Fermi arc surface states, drumhead surface states, chiral zero modes, and quantum oscillations (9-11). These topological states have potential applications ranging from spin electronic devices (12) to quantum information technology (13) .

Recently, the topological properties of higher-dimensional systems ( $\mathrm{n}>3$ ) have drawn great attention, such as 4D quantum Hall effect (14-19), Yang monopoles (20-22), and 2D Weyl surfaces in 5D systems (23-27). Such systems are expected to possess properties that their lowerdimensional counterparts do not support, i.e., those associated with a nonzero second Chern number $\left(\mathrm{C}_{2}\right)$. It has been theoretically verified that (23-25) Weyl surfaces - the higher dimensional extension of the traditional 0D Weyl point that are characterized by a U(1) second Chern number $\mathrm{C}_{2}$, form a Hopf link with a topological linking number equal to $\mathrm{C}_{2}$. However, no realizable systems have been proposed yet. Here, we experimentally demonstrate metamaterials exhibiting linked 2D Weyl surfaces and nontrivial Weyl arcs, as a result of the nontrivial $\mathrm{C}_{2}$. In our systems, in addition to the three-dimensional momentum space, we consider two more synthetic dimensions - two bi-anisotropic terms, as the synthetic fourth and fifth momentum vector components. We investigate the key signatures such as linking between Weyl surfaces in selected 3D subspaces through judicious design of the metamaterials. Furthermore, a 1D Weyl arc on a 3D Fermi hypersurface at the 4D boundary of a 5D system possessing Yang monopoles or Weyl surfaces is highlighted to verify the unique phenomenon protected by $\mathrm{C}_{2}$ in the higher dimensional system.

We start from a 3D photonic system possessing two Dirac points located at $\left[\vec{K}_{D} ; \omega_{D}\right]=$ $\left[0,0, \pm k_{D} ; \omega_{p}\right]$ in the momentum space $(28,29)$, which can be realized by a uniaxial metamaterial with both permittivity and permeability along the axis given by $\varepsilon_{z}=\mu_{z}=1-$ $\omega_{p}^{2} / \omega^{2}$. By introducing a purely asymmetric bianisotropic matrix: $\gamma_{z x}=-\gamma_{x z}, \gamma_{z y}=-\gamma_{y z}$, the Dirac point can be promoted into a 5D-Yang monopole (20) with the effective Hamiltonian [see section I in (30)]:

$$
H_{Y}=\omega_{k 0} \cdot I+\sum_{i=1}^{5} \Phi_{i}(\vec{k}) \cdot \Gamma_{i}
$$

Where $\omega_{k 0}=v_{t} k_{3}, \Phi_{i}(\vec{k})=v_{i} k_{i}, \vec{k}=\left[p_{x}, p_{y}, p_{z}, \omega_{p} \gamma_{x z}, \omega_{p} \gamma_{y z}\right]$ with $\vec{p}=\vec{K}-\vec{K}_{D}, \vec{v}=$ $\left[v_{p}, v_{p}, v_{t}, v_{p}, v_{p}\right]$ determined by the properties of the metamaterial, and $\vec{\Gamma}=$ $\left[-\sigma_{0} \tau_{1}, \sigma_{3} \tau_{2}, \sigma_{0} \tau_{3}, \sigma_{2} \tau_{2}, \sigma_{1} \tau_{2}\right]$ with $\sigma_{i}$ and $\tau_{i}$ being the Pauli matrices. In this perspective, we can consider the 3D Dirac point as the projection of a 5D Yang monopole onto the 3D momentum space. The Hamiltonian $H_{Y}$ satisfies $\boldsymbol{T} \boldsymbol{P}$ symmetry ( $\boldsymbol{T}$ is time-reversal symmetry and $\boldsymbol{P}$ is space-inversion symmetry) with $\boldsymbol{T}=i \sigma_{2} \tau_{0} \boldsymbol{K}(\boldsymbol{K}$ is the complex conjugation $)$ and $(\boldsymbol{T P})^{2}=$ 
-1 , and possesses a globally doubly degenerate band structure $\omega=\omega_{k} \pm|\vec{\Phi}|$, as shown in Fig. 1A. By defining a U(2) Berry connection, one can calculate its Non-Abelian second Chern number $C_{2}^{N A}= \pm 1$.

The Weyl surfaces can be obtained by introducing a $\boldsymbol{P}$ breaking term, which deforms the Yang monopole, as shown in Fig. 1B-C. By introducing a single perturbation term $a \cdot \Gamma_{m n}$ to the original Hamiltonian $H_{Y}$, one obtains,

$$
H_{W S}=\omega_{k 0} \cdot I+\sum_{i=1}^{5} \Phi_{i}(\vec{k}) \cdot \Gamma_{i}+a \cdot \Gamma_{m n}=H_{Y}+a \cdot \Gamma_{m n}
$$

with $\Gamma_{m n}=\frac{i}{2} \cdot\left[\Gamma_{m}, \Gamma_{n}\right](m \neq n \neq 3)$. Here, $k_{3}$ plays a special role due to the presence of term $\omega_{k 0}=v_{t} k_{3}$. The four eigenfrequencies are $\omega(\vec{k})=\omega_{k} \pm \sqrt{|\vec{\Phi}|^{2}+a^{2} \pm 2 a \sqrt{\Phi_{i}^{2}+\Phi_{j}^{2}+\Phi_{3}^{2}}}$, in which $\left[\Phi_{i}, \Phi_{j}, \Phi_{m}, \Phi_{n}, \Phi_{3}\right]$ are the five unsorted momentum vector components. The degeneracy points of these dispersion spectra form two 2-manifolds: an $S^{2}$ ellipsoid $\mathcal{M}_{1}: \Phi_{i}^{2}+$ $\Phi_{j}^{2}+\Phi_{3}^{2}=a^{2}, \Phi_{m}=\Phi_{n}=0$ with $\omega_{\mathcal{M} 1}=\omega_{k 0}$ and a $T^{2}$ plane $\mathcal{M}_{2}: \Phi_{i}=\Phi_{j}=\Phi_{3}=0$ with $\omega_{\mathcal{M} 2}=\omega_{k} \pm \sqrt{\Phi_{\mathrm{m}}^{2}+\Phi_{n}^{2}+a^{2}}$. These degeneracy points can be seen clearly in the dispersion along $k_{3}$ for selected zero/nonzero $k_{m}\left(k_{n}\right)$ and $k_{i}\left(k_{j}\right)$ momentum vector components, as shown in Fig. 1B. The degeneracy $\mathcal{M}_{1}$ points are lifted with nonzero $k_{m}\left(k_{n}\right)$ and the degeneracy $\mathcal{M}_{2}$ points are lifted with nonzero $k_{i}\left(k_{j}\right)$, meaning that $\mathcal{M}_{1}$ and $\mathcal{M}_{2}$ are both 2D manifolds in the 5D space.

The perturbation term $\Gamma_{m n}$ for different combinations of $m$ and $n$ in Eq. (2) can be realized by introducing specific types of symmetry breaking terms into the Yang monopole system [see section I in (30)]. It should be noted that different choices of $[m, n]$ only rotate the Weyl surfaces' orientation in the 5D space but do not affect the system's overall topological property. Indeed, the Weyl surfaces described by Eq. (2) always consist of three 2-fold degeneracy nodal surfaces, and each point on the nodal surfaces serves as a Weyl point in the corresponding 3codimensions subspace orthogonal to the nodal surface [See Section II in (30)] . Importantly, this selective rotation can help us study the key characteristics of $H_{W S}$ in the momentum space, by avoiding the difficulty of investigating the system in the synthetic dimensions $k_{4}$ and $k_{5}$, which are fixed for a given metamaterial design. As shown in Fig. 1D-F, in the 3D momentum space, for the choice of perturbation along $\Gamma_{45}$, the Weyl surfaces appear as two overlapped Weyl points wrapped by a degeneracy $S^{2}$ ellipsoid; while with the choice of the perturbation along $\Gamma_{15}$, the Weyl surfaces appear as linked nodal lines; with the choice of the perturbation along $\Gamma_{12}$, the Weyl surfaces take the form of two Weyl points located on both sides of a degeneracy $T^{2}$ plane. Interestingly, the appearance of the Weyl surfaces in the 3D subspace formed by $k_{4}, k_{2}$ and $k_{3}$ in a system with $\Delta H=a \cdot \Gamma_{45}$ is the same as that of a system with $\Delta H=a \cdot \Gamma_{15}$ in the 3D real momentum space. This correspondence indicates that the above configurations (Fig. 1D-F) in the $3 \mathrm{D}$ momentum space with different choices of $[m, n]$ just correspond to the different intersections of the same Weyl surfaces in the 5D space. Note that the Abelian second Chern number $C_{2}^{A}$ of these 2-manifold Weyl surfaces is the same as the Non-abelian one in the original Yang monopole system: $\left.C_{2}^{A}\right|_{W S}=\left.C_{2}^{N A}\right|_{Y M}= \pm 1$. This global topological invariant describes the 
linking number of these two Weyl surfaces $(24,25)$, which corresponds to the nodal link shown in Fig. 1E with two dimensions hidden [See Section III in (30)].

The designs of metamaterials possessing Weyl surfaces corresponding to different perturbation terms $\Gamma_{m n}$ are provided in section IV in (30). Here we experimentally realize the sample in Fig. S2E, which corresponds to $\Delta H \approx a \cdot \Gamma_{15}$, because it reveals the key topological feature of linking between the two Weyl surfaces. The projected bulk states (PBS) of this system are probed by using the transmitted nearfield scanning configuration described in section VII in (30). The PBS exhibits very distinct features in the three different frequency regions (FR) divided by the two longitudinal modes (the two flat lines in the left panel of Fig. 1B), located at about $7.77 \mathrm{GHz}$ and $8.06 \mathrm{GHz}$ from the simulation data: regions I, II, and III correspond to frequency ranges below, between, and above the two longitudinal modes, respectively (marked with different colors in Fig. 1B). At the lowest frequency range FR-I, the outline of the PBS appears as two intersecting hyperbolic curves, as clearly shown by the effective medium theory (EMT) and full-wave simulation (Fig. 2A). With the increase of frequency into region FR-II, the PBS transforms into a hyperbolic curve and an elliptic curve intersecting each other, with an empty density of states inside the overlapped eye-shaped region (Fig. 2B). With further increase of the frequency into region FR-III, the outline of the PBS turns into two intersecting elliptical curves (Fig. 2C). The symmetry operators $\Gamma_{1} \Gamma_{5}$ and $\Gamma_{2} \Gamma_{4}$ guarantee that the intersections occur on the plane $k_{x}=0$ in FR-II and $k_{y}=0$ in FR-I and FR-III, respectively. By identifying the intersection points in both simulation and EMT results, the locations of the 5D Weyl surfaces in the momentum subspace are obtained and plotted in Fig. 2D. The two lines projected by $\mathcal{M}_{2}$ thread through the red circles projected by $\mathcal{M}_{1}$, in both the simulation and the EMT. This nonzero linking number between the projected $\mathcal{M}_{1}$ and $\mathcal{M}_{2}$ Weyl surfaces reveals the nontrivial $C_{2}$ of the Weyl surfaces in the 5D space. In FR-I and FR-II, pronounced surface states are also observed in both EMT and experimental results, which correspond to the drumhead surface states typically present in nodal line systems. It is worth mentioning that, from a 5D perspective, the drumhead surface states are actually a special cross-section of the Fermi hypersurface. Note that due to the limited spatial resolution in the momentum space in measurement (arising from the finite size of the sample), the intersecting points cannot be clearly identified in the measured PBS. Nonetheless, the good match in the outlines of the PBS and the drumhead surface states between the measurement and the simulation serves as strong evidence of the presence of linked nodal surfaces in the fabricated sample.

Besides the linking of the Weyl surfaces, the nontrivial $C_{2}$ in a $5 \mathrm{D}$ system is also manifested by the presence of 3D Fermi hypersurfaces and 1D Weyl arcs at the 4D boundaries of 5D nontrivial systems, which has been theoretically investigated in detail (25). Our effective medium model shows the presence of Fermi hypersurfaces and surface Weyl points (crossing points on the hypersurface) in both Yang monopole and Weyl surface systems, as shown in Fig. $3 \mathrm{~A}-\mathrm{B}$, where the Fermi hypersurface is formed by the evolution of the Fermi arcs with the synthetic dimensions, and the surface Weyl points correspond to the crossing points on the 3D Fermi hypersurface. The surface Weyl points at continuously varying frequencies form a 1D Weyl arc, as shown in Fig. 3C and 3D. In comparison to the Yang monopole system, the medium with linked Weyl surfaces has asymmetric Weyl arcs with respect to the $k_{y}=0$ plane. For the Yang monopole case, by enforcing additional symmetry operators $\Gamma_{2} \Gamma_{4}$ and $\Gamma_{2} \Gamma_{5}$ and the impedance matching condition with the surrounding medium, the Weyl arc node can be 
arcs described above for both the Yang monopole and Weyl surfaces represent a unique phenomenon arising from the nontrivial $C_{2}$.

For experimental observation of the Weyl arcs, we consider a metamaterial with Yang monopoles, whose configuration is shown in Fig. S2A. The measured Fermi arc states shown in Fig. 3E-F are in good agreement with both EMT and CST simulated results. However, at the points (blue circles) where the surface Weyl nodes are expected, a small gap is observed in Fig. $3 \mathrm{~F}$. This gap closes at the Yang monopole near $7.95 \mathrm{GHz}$, and gradually increases away from this frequency, and eventually disappears into the light cone. The origin of this gap is due to the impedance mismatch with the surrounding medium - air. However, the Weyl arcs, protected by the nonzero $C_{2}$, is guaranteed to exist in the 4D synthetic space of the boundary $\left(k_{y}, k_{z}, k_{4}\right.$ and $k_{5}$ in our configuration) despite this impedance mismatch. [see Section V\&VI in (30) for numerical verification].

We have established an effective medium based frame to realize the linked Weyl surfaces and intriguing surface states due to the nontrivial $\mathrm{C}_{2}$ of the systems. By using bi-anisotropic terms as the synthetic fourth/fifth dimensions, and by further introducing specific $\boldsymbol{P}$-breaking terms to deform the Yang monopole, the medium offers the possibility to systematically study the linked 5D Weyl surface system. Our work provides a unique platform to explore the topological properties of complicated electromagnetic systems in higher dimensions.

\section{References and Notes}

1. A. A. Burkov, Nat. Mater. 15, 1145-1148 (2016).

2. A. H. Castro Neto, F. Guinea, N. M. R. Peres, K. S. Novoselov, A. K. Geim, Rev. Mod. Phys. 81, 109-162 (2009).

3. C. L. Kane and E. J. Mele, Phys. Rev. Lett. 95, 226801 (2005)

4. X. Wan, A. M. Turner, A. Vishwanath, S. Y. Savrasov, Phys. Rev. B 83, 205101 (2011)

5. S. Y. Xu et al, Science 349, 613 (2015)

6. L Lu et al., Science 349, 622 (2015)

7. B. Q. Lv et al., Phys. Rev. X 5, 031013 (2015).

8. B. Yang et al., Science 359, 1013-1016 (2018).

9. N. P. Armitage, E. J. Mele, A. Vishwanath, Rev. Mod. Phys. 90, 015001 (2018).

10. T. Ozawa et al., Rev. Mod. Phys. 91, 015006 (2019).

11. M. Kim, Z. Jacob, J. Rho, Light Sci. Appl. 9, 130 (2020).

12. M. I. Shalaev, W. Walasik, A. Tsukernik, Y. Xu, N. M. Litchinitser, Nat. Nanotechnol. 14, 31-34 (2019).

13. N. E. Bonesteel, L. Hormozi, G. Zikos, S. H. Simon, Phys. Rev. Lett. 95, 140503 (2005).

14. S. C. Zhang, J. Hu, Science 294, 823-828 (2001).

15. X. L. Qi, T. L. Hughes, S. C. Zhang, Phys. Rev. B 78, 195424 (2008).

16. H. M. Price, O. Zilberberg, T. Ozawa, I. Carusotto, N. Goldman, Phys. Rev. Lett. 115, 195303 (2015).

17. M. Lohse, C. Schweizer, H. M. Price, O. Zilberberg, I. Bloch, Nature 553, 55-58 (2018).

18. R. Yu, Y. X. Zhao, A. P. Schnyder, Natl. Sci. Rev. 7, 1288-1295 (2020).

19. O. Zilberberg et al., Nature 553, 59-62 (2018).

20. C. N. Yang, J. Phys. A Math. Theor. 19, 320-328 (1978).

21. S. Sugawa, F. Salces-Carcoba, A. R. Perry, Y. Yue, I. B. Spielman, Science 360, 14291434 (2018). 
22. $\quad$ K. Hasebe, Nucl. Phys. B 956, 115012 (2020).

23. B. Lian, S. C. Zhang, Phys. Rev. B 94, 041105 (2016).

24. B. Lian, S. C. Zhang, Phys. Rev. B 95, 235106 (2017).

25. J. Y. Chen, B. Lian, S. C. Zhang, Phys. Rev. B 100, 075112 (2019).

26. K. Hashimoto, X. Wu, T. Kimura, Phys. Rev. B 95, 165443 (2017).

27. K. Hashimoto, Y. Matsuo, Phys. Rev. B 101, 245138 (2020).

28. Q. Guo et al., Phys. Rev. Lett. 119, 213901 (2017).

29. Q. Guo et al., Phys. Rev. Lett. 122, 203903 (2019).

30. Materials and methods are available as supplementary materials.

Acknowledgments:

Funding: This work was supported in part by the Horizon 2020 Action Project Nos. 648783 (TOPOLOGICAL), 734578 (D-SPA), and 777714 (NOCTORNO); the Engineering and Physical Sciences Research Council (EP/J018473/1).

Author contributions: S.M. performed numerical simulations and analytical calculations, Y. B. and O.Y. conducted the experiment, B.Y., Q.G., J.F., and H.S. participated in the analysis of the results, and S.Z. supervised the project. ${ }^{\dagger}$ S. M. and Y. B. contributed equally to this work.

Competing interests: The authors declare no conflict of interest.

Data and materials availability: All data are available in the main text or the supplementary materials.

\section{Supplementary Materials}

Supplementary Text

Figs. S1 to S14

References 

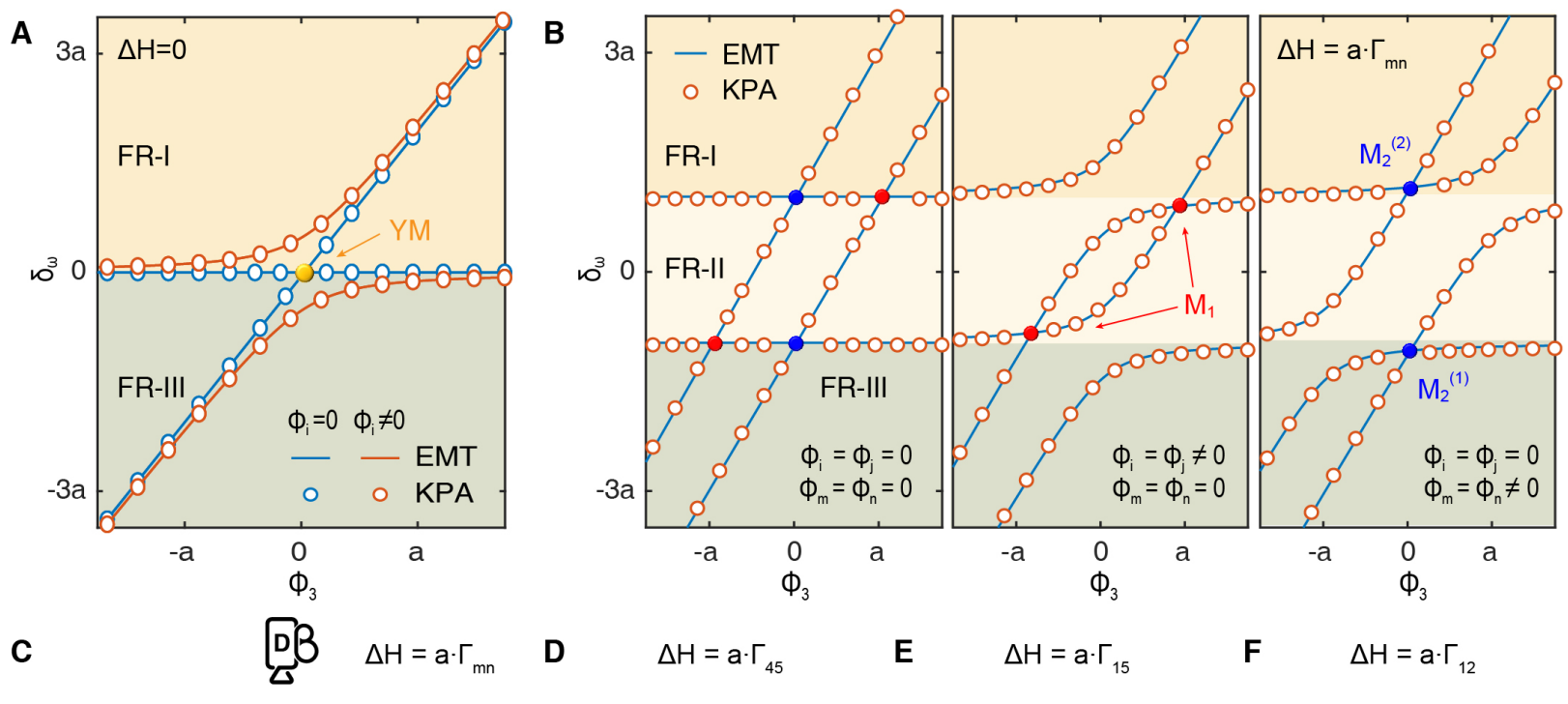

D $\quad \Delta \mathrm{H}=\mathrm{a} \cdot \Gamma_{45}$

E $\quad \Delta \mathrm{H}=\mathrm{a} \cdot \Gamma_{15}$

$\mathbf{F} \quad \Delta \mathrm{H}=\mathrm{a} \cdot \Gamma_{12}$
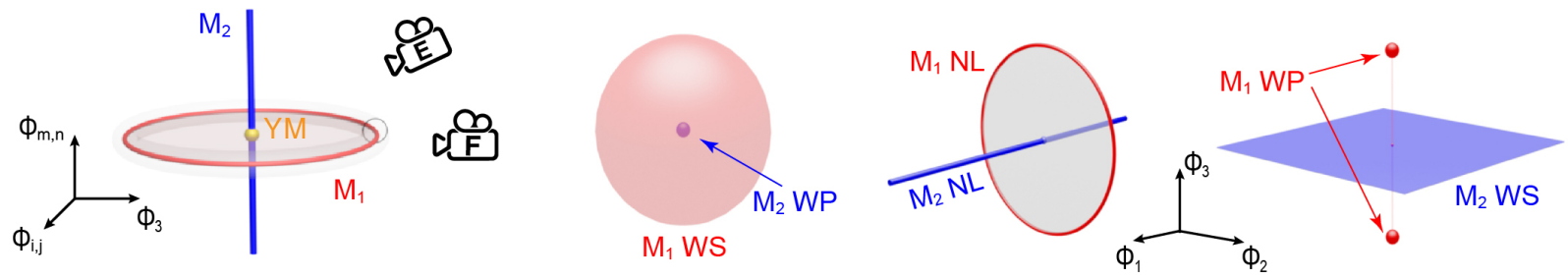

Fig. 1. Illustration of Weyl surfaces in 5D space. The dispersion spectrum along $k_{3}$ for (A) the Yang monopole system and (B) the general Weyl surface system $H=H_{Y}+a \cdot \Gamma_{m n}$, for various $\Phi_{m}\left(\Phi_{n}\right)$ and $\Phi_{i}\left(\Phi_{j}\right)$. The colored areas represent different frequency regions FR-I to FR-III divided by the longitudinal modes. (C) 5D k-space distribution of the Yang monopole and the linked Weyl surfaces. (D-F) Three representations of Weyl surfaces in the 3D real momentum subspace, with (D) $\Delta H=a \cdot \Gamma_{45}$, (E) $\Delta H=a \cdot \Gamma_{15}$ and (F) $\Delta H=a \cdot \Gamma_{12}$. 


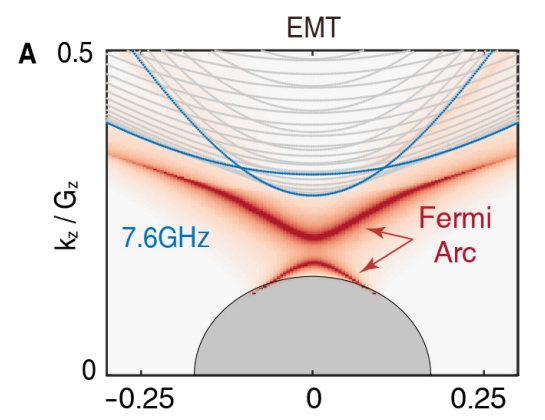

$k_{x} / G_{x}$
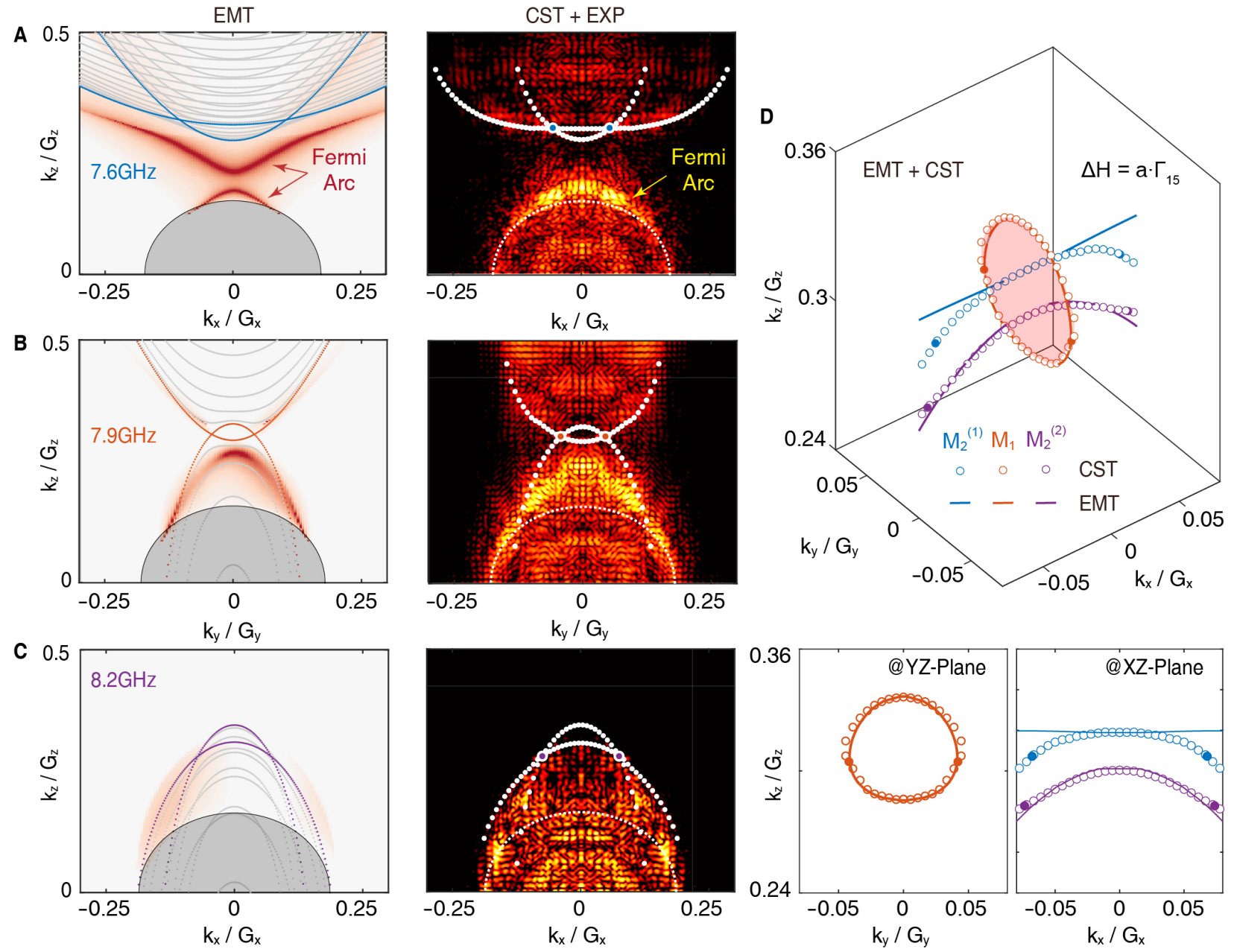

Fig. 2. Simulation and characterization of metamaterials exhibiting linked Weyl surfaces with $\Delta \boldsymbol{H} \approx \boldsymbol{a} \cdot \boldsymbol{\Gamma}_{\mathbf{1 5}}$. (A-C) The simulated and measured PBS at three different frequencies in the three regimes of FR-I, FR-II, and FR-III, respectively. The grey area represents the light cone. (D) The location of the Weyl surfaces projected in the three-dimensional real momentum space, with solid circles representing the selected frequency in (A-C). 
A
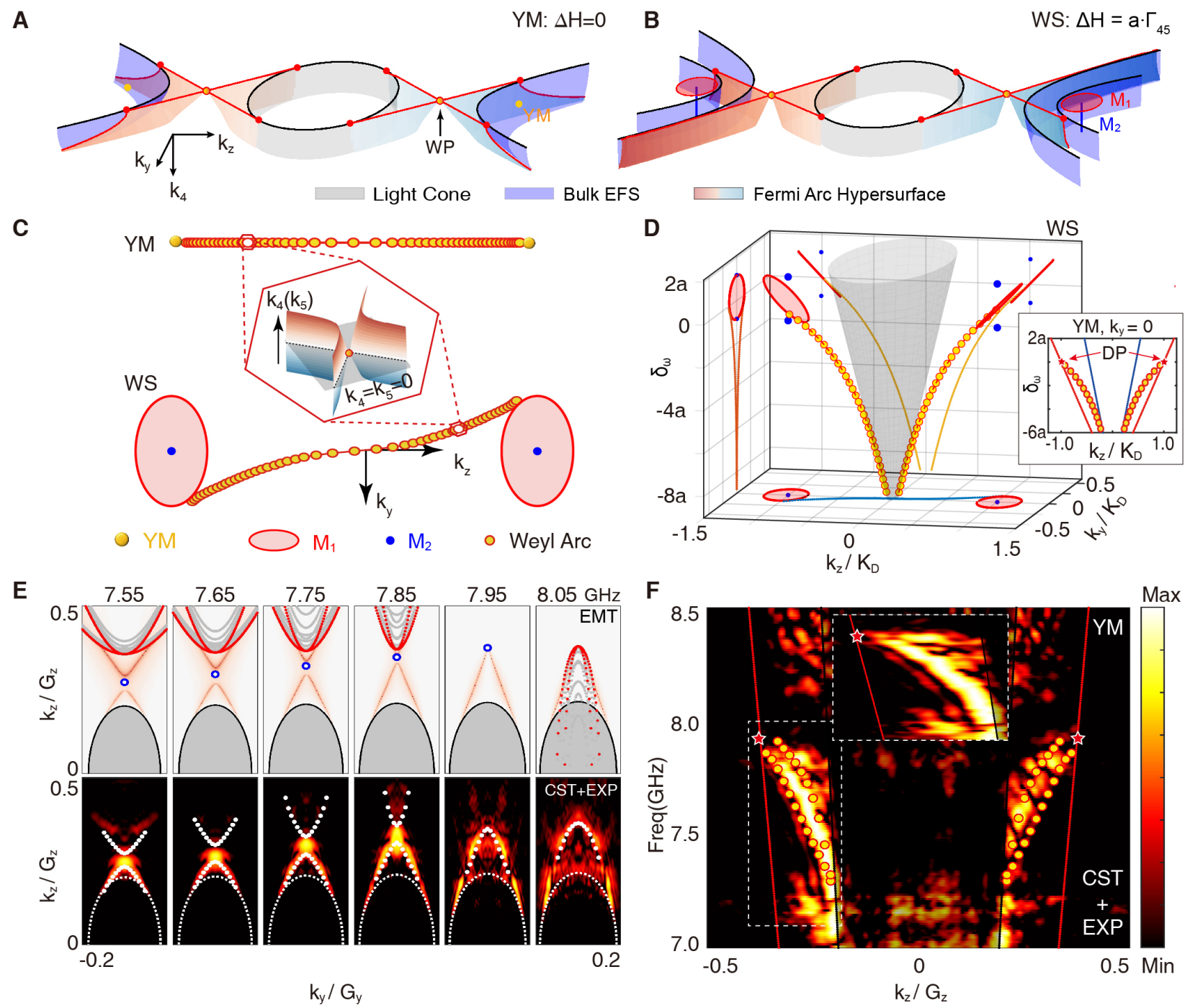

Fig. 3. The boundary states of metamaterials possessing Yang monopole and Weyl surfaces. (A, B) Illustration of the Fermi hypersurface and surface Weyl point for both (A) Yang monopole and (B) Weyl surface with $\Delta H=a \cdot \Gamma_{45}$, with one dimension $k_{5}$ hidden. The yellows spheres in (A) represent the Yang monopoles, and the red circles and blue lines in (B) represent the cross-sections of different Weyl surfaces, respectively. (C) The 1D Weyl arcs for both a system with Yang monopole and that with Weyl surfaces. (D) The dispersion of the 1D Weyl arc in the Weyl surface system and its projection in all three directions, with the counterpart of the Yang monopole case shown in the inset. (E) The numerical and measured bulk/surface states for the Yang monopole system. The numerical results are retrieved by both EMTs and CST simulations. The blue circles in the upper panel represent the projection of Weyl arc points calculated by EMT. (F) The simulated and measured dispersion of surface states along $k_{z}$ direction. The small gap arises from the impedance mismatch, which implies a slight shift of the Weyl arcs away from $k_{z}$ axis. 


\section{Science МIAAAS}

\section{Supplementary Materials for}

Linked Weyl Surfaces and Weyl Arcs in Photonic Metamaterials

Shaojie Ma, Yangang Bi, Qinghua Guo, Biao Yang, Oubo You, Jing Feng, Hong-Bo Sun, Shuang Zhang*

Correspondence to: shuzhang@hku.hk

This PDF file includes:

Supplementary Text

Figs. S1 to S14

References 


\section{Supplementary Text}

\section{$\underline{\text { I. Derivation of effective Hamiltonian } \underline{\text { using the }} \underline{\boldsymbol{k} \cdot \boldsymbol{p}} \text { approximation }}$}

In this section, we describe how to start from the effective medium theory (EMT) model to derive the effective Hamiltonian using the $k \cdot p$ approximation (KPA) method.

\section{A. Derivation}

A general electromagnetic medium satisfies the constitutive equations: $\vec{D}=\varepsilon_{0} \varepsilon \cdot \vec{E}+i \gamma \cdot \vec{H} \& \vec{B}=$ $\mu_{0} \boldsymbol{\mu} \cdot \vec{H}+(i \gamma)^{\dagger} \cdot \vec{E}$. For a medium slightly deviating from the uniaxial metamaterial that behaves like a 3D Dirac point (DP) shown in Ref. $(28,29)$, the corresponding electromagnetic tensor elements are expressed as:

$$
\vec{\varepsilon}=\left[\begin{array}{ccc}
\varepsilon_{p} & i \chi & 0 \\
-i \chi & \varepsilon_{p}+\delta_{\varepsilon} & 0 \\
0 & 0 & \varepsilon_{t}
\end{array}\right], \vec{\mu}=\left[\begin{array}{ccc}
\mu_{p} & 0 & 0 \\
0 & \mu_{p} & 0 \\
0 & 0 & \mu_{t}
\end{array}\right], \vec{\gamma}=\left[\begin{array}{ccc}
\gamma_{x x} & 0 & \gamma_{x z} \\
0 & \gamma_{y y} & \gamma_{y z} \\
\gamma_{z x} & \gamma_{z y} & \gamma_{z z}
\end{array}\right] .
$$

Note that for a medium possessing DP, it only contains the main terms $\varepsilon_{p}, \mu_{p}, \varepsilon_{t}, \mu_{t}$ which satisfies a perfect electromagnetic duality with $\varepsilon_{p} / \mu_{p}=\varepsilon_{t} / \mu_{t}$ and $\varepsilon_{t} / \varepsilon_{t 0}=\mu_{t} / \mu_{t 0}=1-\omega_{p}{ }^{2} / \omega^{2}$. Weyl surfaces can result from the additional perturbation terms in Eq. (S1), i.e. the magneto-optic effect $\chi$, the anisotropic term $\delta_{\varepsilon}$, the non-degeneracy term $\delta_{p}$ with unequal electric and magnetic bulk plasmon frequencies, i.e. $\omega_{p E}=\omega_{p} \cdot\left(1-\delta_{p}\right)$ and $\omega_{p H}=\omega_{p} \cdot\left(1+\delta_{p}\right)$, and the bi-anisotropic and the chiral terms shown in the matrix $\vec{\gamma}$.

For the DP medium, by using the method shown in Ref. $(8,28)$, and by introducing some auxiliary parameters $P_{z}, J_{p z}$ and $M_{z}, J_{m z}$ to linearize the standard Maxwell's equations, we obtain:

$$
\begin{aligned}
& P_{z}=-\frac{\varepsilon_{0} \varepsilon_{t 0} \omega_{p E}^{2}}{\omega^{2}} E_{z} \Rightarrow \varepsilon_{0} \varepsilon_{t 0} \omega_{p E}^{2} E_{z}=-\omega^{2} P_{z}=-i \omega J_{z} \& J_{p z}=-i \omega P_{z} \\
& M_{z}=-\frac{\mu_{0} \mu_{t 0} \omega_{p H}{ }^{2}}{\omega^{2}} H_{z} \Rightarrow \mu_{0} \mu_{t 0} \omega_{p H}^{2} H_{z}=-\omega^{2} M_{z}=-i \omega J_{m z} \& J_{m z}=-i \omega M_{z}
\end{aligned}
$$

An 8-Dimension vector $\Phi=\left[E_{x}, E_{y}, E_{z}, H_{x}, H_{y}, H_{z}, J_{p z}, J_{m z}\right]^{T}$ can be used to represent the eigenvector, and by assuming a steady-state with all fields varying as $\exp (-i \omega t)$, the Maxwell equations can be rewritten as an intrinsic matrix equation for $\omega: R_{0} \widetilde{\Psi}_{0}=\omega S_{0} \widetilde{\Psi}_{0}$. By solving the matrix directly, the fourfold degeneracy Dirac points can be obtained, which are located at $\left[\vec{K}_{D}, \omega_{D}\right]=\left[0,0, \pm k_{D} ; \omega_{p}\right]$ with $k_{D}=n_{p} \cdot \omega_{p} / c$ and $n_{p}=\sqrt{\varepsilon_{p} \mu_{p}}$.

The Dirac point located at $k_{z}=k_{D}$ possesses eigenfunctions contained in the following matrix: 


$$
\widetilde{\Psi}_{\text {Dirac }}=\frac{1}{2} \cdot \operatorname{diag}\left[\begin{array}{c}
\varepsilon_{0} \varepsilon_{p} \\
\varepsilon_{0} \varepsilon_{p} \\
\varepsilon_{0} \varepsilon_{t} \\
\mu_{0} \mu_{p} \\
\mu_{0} \mu_{p} \\
\mu_{0} \mu_{t} \\
1 / \varepsilon_{0} \varepsilon_{t} \omega_{p}{ }^{2} \\
1 / \mu_{0} \mu_{t} \omega_{p}{ }^{2}
\end{array}\right]^{-1 / 2} \cdot\left[\begin{array}{cccc}
1 & 0 & 1 & 0 \\
-i & 0 & i & 0 \\
0 & 1 & 0 & 1 \\
i & 0 & -i & 0 \\
1 & 0 & 1 & 0 \\
0 & i & 0 & -i \\
0 & i & 0 & i \\
0 & -1 & 0 & 1
\end{array}\right] \text {. }
$$

These eigenmodes correspond to polarization states $\left[R C P, E_{z}+i H_{z}, L C P, E_{z}-i H_{z}\right]$, respectively.

A similar Linearization process is used to obtain the intrinsic matrix equation at the momentum $\vec{K}=\vec{K}_{D}+\vec{p}$ for the perturbed WS medium, that is $R \widetilde{\Psi}=\omega S \widetilde{\Psi}$, with:

$$
\vec{R}=\left[\begin{array}{cccc}
0 & -\vec{K}_{c} & -i \cdot \vec{I}_{(3,1)} & 0 \\
\vec{K}_{c} & 0 & 0 & -i \cdot \vec{I}_{(3,1)} \\
\vec{i}_{(1,3)} & 0 & 0 & 0 \\
0 & i \vec{I}_{(1,3)} & 0 & 0
\end{array}\right], \vec{S}=\left[\begin{array}{cccc}
\varepsilon_{0} \vec{\varepsilon}_{N} & i \vec{\gamma} & 0 & 0 \\
(i \vec{\gamma})^{+} & \mu_{0} \vec{\mu}_{N} & 0 & 0 \\
0 & 0 & \left(\varepsilon_{0} \varepsilon_{t 0} \omega_{p E}\right)^{-1} & 0 \\
0 & 0 & 0 & \left(\mu_{0} \mu_{t 0} \omega_{p H}{ }^{2}\right)^{-1}
\end{array}\right]
$$

In these block matrices, we define:

$$
\vec{K}_{c}=\left[\begin{array}{ccc}
0 & -k_{z} & k_{y} \\
k_{z} & 0 & -k_{x} \\
-k_{y} & k_{x} & 0
\end{array}\right] \& \vec{I}_{(3,1)}=\vec{I}_{(1,3)}^{\dagger}=\left[\begin{array}{l}
0 \\
0 \\
1
\end{array}\right],
$$

and the corresponding nondispersive permittivity/permeability matrices read:

$$
\vec{\varepsilon}_{N}=\left[\begin{array}{ccc}
\varepsilon_{p} & i \chi & 0 \\
-i \chi & \varepsilon_{p}+\delta_{\varepsilon} & 0 \\
0 & 0 & \varepsilon_{t 0}
\end{array}\right], \vec{\mu}_{N}=\left[\begin{array}{ccc}
\mu_{p} & 0 & 0 \\
0 & \mu_{p} & 0 \\
0 & 0 & \mu_{t 0}
\end{array}\right] .
$$

Using the DP medium's eigenmodes as the basis and expand the WS medium's intrinsic matrix, we can obtain the KPA-Hamiltonian:

$$
\left.\begin{array}{l}
R_{K P A}=\widetilde{\Psi}_{\text {Dirac }}^{\dagger} \cdot R \cdot \widetilde{\Psi}_{\text {Dirac }} \& S_{K P A}=\widetilde{\Psi}_{\text {Dirac }}^{\dagger} \cdot S \cdot \widetilde{\Psi}_{\text {Dirac }} \\
R \cdot \widehat{\Psi}_{j}=\omega_{j} \cdot S \cdot \widehat{\Psi}_{j} \& \widehat{\Psi}_{j}=\widetilde{\Psi}_{\text {Dirac }, i} \Psi_{i j}
\end{array}\right\} \Rightarrow R_{K P A} \cdot \Psi=\omega \cdot S_{K P A} \cdot \Psi,
$$

In the perturbed case with $R_{K P A}=\omega_{p} \cdot I+\delta R_{K P A}, S_{K P A}=I+\delta S_{K P A}, \omega=\omega_{p}+\delta \omega$, the effective KPA Hamiltonian which satisfies $H_{K P A} \Psi=\delta \omega \cdot \Psi$ can be written as: $H_{K P A}=\delta R_{K P A}-\omega_{p} \cdot \delta S_{K P A}$.

For the sake of simplicity, we only write the results based on the Dirac matrix, that is:

$$
\boldsymbol{H}=\sum_{i=1}^{5} \Phi_{i} \cdot \boldsymbol{\Gamma}_{\boldsymbol{i}}+\sum_{s} a_{s} \cdot \boldsymbol{\Gamma}_{\boldsymbol{s}}+\Phi_{0} \cdot I
$$

In this equation, $\boldsymbol{\Gamma}_{\boldsymbol{i}}=\left[-\sigma_{0} \sigma_{1}, \sigma_{3} \sigma_{2}, \sigma_{0} \sigma_{3}, \sigma_{2} \sigma_{2}, \sigma_{1} \sigma_{2}\right], \Phi_{i}=v_{i} k_{i}$ in which:

$$
\vec{v}=\left[v_{p}, v_{p}, v_{t}, v_{p}, v_{p}\right] \text {, and } \vec{k}=\left[p_{x}, p_{y}, p_{z}-\frac{\delta_{\varepsilon} \omega_{p}}{4 c} \cdot \sqrt{\frac{\mu_{p}}{\varepsilon_{p}}}, \frac{1}{2} \omega_{p} \cdot\left(\gamma_{x z}-\gamma_{z x}\right), \frac{1}{2} \omega_{p} \cdot\left(\gamma_{y z}-\gamma_{z y}\right)\right]
$$


where $v_{p}=\frac{c}{2 \sqrt{\varepsilon_{t} \mu_{p}}}, v_{t}=\frac{c}{2 n_{p}}, \vec{p}=\vec{K}-\vec{K}_{D}$ and $\Phi_{0}=\Phi_{3}$. Besides, the equation has some additional perturbation terms $\Delta H=\sum_{s} a_{s} \boldsymbol{\Gamma}_{s}$, which deforms the Yang monopole Hamiltonian, and it can be divided into the following three parts:

$$
\begin{gathered}
\Delta H_{1}=-\frac{\delta_{\varepsilon} \omega_{p}}{4 \varepsilon_{p}} \cdot \frac{\Gamma_{24}+\Gamma_{15}}{2}+\delta_{p} \omega_{p} \cdot \frac{\Gamma_{24}-\Gamma_{15}}{2} \\
\Delta H_{2}=\left[\frac{\chi \omega_{p}}{2 \varepsilon_{p}}-\frac{c \omega_{p}}{2 n_{p}} \cdot\left(\gamma_{x x}+\gamma_{y y}\right)\right] \cdot \frac{\Gamma_{12}+\Gamma_{45}}{2}+\frac{c \gamma_{z z} \omega_{p}}{2 \varepsilon_{t} \sqrt{\mu_{p} / \varepsilon_{p}}} \cdot \frac{\Gamma_{12}-\Gamma_{45}}{2} \\
\Delta H_{3}=\frac{1}{2} v_{p} \omega_{p} \cdot\left(\gamma_{x z}+\gamma_{z x}\right) \cdot \Gamma_{23}+\frac{1}{2} v_{p} \omega_{p} \cdot\left(\gamma_{y z}+\gamma_{z y}\right) \cdot \Gamma_{13}
\end{gathered}
$$

Here, $\Gamma_{m n}=\frac{i}{2} \cdot\left[\Gamma_{m}, \Gamma_{n}\right](m \neq n)$. This formula satisfies the general Hamiltonian for a WS medium.

A similar process can be used for the WS near the DP located at $k_{z}=-k_{D}$. The result is similar but with some minor differences:

$$
k_{3}=-p_{z}-\frac{\delta_{\varepsilon} \omega_{p}}{4 c} \cdot \sqrt{\frac{\mu_{p}}{\varepsilon_{p}}} \& \Delta H_{2}=\left[\frac{\chi \omega_{p}}{2 \varepsilon_{p}}+\frac{c \omega_{p}}{2 n_{p}} \cdot\left(\gamma_{x x}+\gamma_{y y}\right)\right] \cdot \frac{\Gamma_{12}+\Gamma_{45}}{2}-\frac{c \gamma_{z z} \omega_{p}}{2 \varepsilon_{t} \sqrt{\mu_{p} / \varepsilon_{p}}} \cdot \frac{\Gamma_{12}-\Gamma_{45}}{2}
$$

With the complete mapping between the effective medium description and the effective Hamiltonian, as shown above, we can discuss the Weyl surface in detail. Remarkably, adjusting the ratio between the corresponding parameters $\left[\gamma_{x x}+\gamma_{y y}, \gamma_{z z}\right]$ and $\left[\delta_{\varepsilon}, \delta_{p}\right]$ can lead to a particular $\Gamma_{m n}$ perturbation term. For example, a pure $\Gamma_{45}\left(\Gamma_{12}\right)$ term results from $\varepsilon_{p} \cdot \gamma_{z z}= \pm \varepsilon_{t} \cdot\left(\gamma_{x x}+\gamma_{y y}\right)$, while the $\Gamma_{15}\left(\Gamma_{24}\right)$ term results from $\delta_{\varepsilon}= \pm 4 \varepsilon_{p} \delta_{p}$, which corresponds to the metamaterial presented in Fig. S2.

It is worth mentioning that for the Weyl surface Hamiltonian $H_{W S}=H_{Y}+a \cdot \Gamma_{m n}$ discussed in the main text, with $\left[k_{i}, k_{j}, k_{m}, k_{n}, k_{3}\right]$ the five unsorted momentum vector components, satisfies the symmetry $\quad\left(\Gamma_{i} \Gamma_{j}\right)^{\dagger} \cdot H_{W S}\left(k_{i}, k_{j}, k_{m}, k_{n}, k_{3}\right) \cdot\left(\Gamma_{i} \Gamma_{j}\right)=H_{W S}\left(-k_{i},-k_{j}, k_{m}, k_{n}, k_{3}\right) \quad$ and $\quad\left(\Gamma_{m} \Gamma_{n}\right)^{\dagger}$. $H_{W S}\left(k_{i}, k_{j}, k_{m}, k_{n}, k_{3}\right) \cdot\left(\Gamma_{m} \Gamma_{n}\right)=H_{W S}\left(k_{i}, k_{j},-k_{m},-k_{n}, k_{3}\right)$, which enable us to see special features in a lower-dimensional subspace.

\section{B. Discussion of the Lorentz term}

In general, the resonance can have a Lorentz term, which will require additional adjustments to the derivation of the effective Hamiltonian. The general electric/magnetic responses with Lorenz resonance are given by:

$$
\varepsilon_{t}=\varepsilon_{t 0}+\frac{g_{1} \cdot \omega_{0}^{2}}{\omega_{0}^{2}-\omega^{2}} \text { and } \mu_{t}=\mu_{t 0}+\frac{g_{2} \cdot \omega^{2}}{\omega_{0}^{2}-\omega^{2}}
$$


, where $\omega_{0}$ is the Lorenz resonance frequency. In Eq. (S12), $g_{1}$ and $g_{2}$ parameters can be chosen to set the longitudinal electric and longitudinal magnetic modes to specific frequencies $\omega_{p E}$ and $\omega_{p H}$, respectively. Under this condition, the permittivity and permeability can be expressed as:

$\varepsilon_{z Z}=\varepsilon_{t 0}\left(1-\frac{\omega_{p E}{ }^{2}-\omega_{0}^{2}}{\omega^{2}-\omega_{0}^{2}}\right) \& \mu_{z Z}=\mu_{t 0} \cdot\left[1-\frac{\omega^{2} \cdot\left(\omega_{p H}{ }^{2}-\omega_{0}^{2}\right)}{\omega_{p H}{ }^{2} \cdot\left(\omega^{2}-\omega_{0}^{2}\right)}\right] \approx \frac{\mu_{t 0} \omega_{0}^{2}}{\omega_{p}^{2}} \cdot\left(1-\frac{\omega_{p H}{ }^{2}-\omega_{0}{ }^{2}}{\omega^{2}-\omega_{0}{ }^{2}}\right)$

These dispersive parameters can be linearized by introducing some auxiliary parameters $P_{z}, J_{p z}$ and $M_{z}, J_{m z}$ to the standard Maxwell's equations (8), and the KPA process can be done similarly.

Here, we define some auxiliary parameters $\beta=\omega_{p} / \omega_{0}, \quad \alpha=\sqrt{1-1 / \beta^{2}}$ and $\kappa=\beta$. $\sqrt{\varepsilon_{t 0} \mu_{p} / \varepsilon_{p} \mu_{t 0}}$. For the WS-medium near the DP with $k_{z}= \pm k_{D}$, the results between the case of the Drude model $\left(\omega_{0}=0\right)$ and that of the Lorentz model $\left(\omega_{0} \neq 0\right)$ are similar, with the comparison presented in the table below.

\begin{tabular}{|c|c|}
\hline$\omega_{0}=0$ & $\omega_{0} \neq 0$ \\
\hline$v_{p}=\frac{c}{2 \sqrt{\varepsilon_{t} \mu_{p}}}$ & $v_{p}=\frac{\alpha \cdot c}{2 \sqrt{\varepsilon_{t} \mu_{p}}}$ \\
\hline$\Phi_{3}= \pm \frac{1}{2 n_{p}} c p_{z}-\frac{\delta_{\varepsilon} \omega_{p}}{8 \varepsilon_{p}}$ & $\Phi_{3}= \pm \frac{1}{2 n_{p}} c p_{z}-\frac{\delta_{\varepsilon} \omega_{p}}{8 \varepsilon_{p}}+\frac{\kappa^{2}-1}{\kappa^{2}+1} \cdot \delta_{p} \omega_{p}$ \\
\hline$\Phi_{0}=\Phi_{3}$ & $\Phi_{0}=\Phi_{3}-\frac{\kappa^{2}-1}{\kappa^{2}+1} \cdot \delta_{p} \omega_{p}$ \\
\hline $\begin{aligned} \Delta H_{1}= & -\frac{\delta_{\varepsilon} \omega_{p}}{4 \varepsilon_{p}} \cdot \frac{\Gamma_{24}+\Gamma_{15}}{2} \\
& +\delta_{p} \omega_{p} \cdot \frac{\Gamma_{24}-\Gamma_{15}}{2}\end{aligned}$ & $\begin{aligned} \Delta H_{1}= & -\frac{\delta_{\varepsilon} \omega_{p}}{2\left(1+\kappa^{2}\right) \varepsilon_{p}} \cdot \frac{\Gamma_{24}+\Gamma_{15}}{2} \\
& +\frac{2 \delta_{p} \omega_{p}}{1+\kappa^{2}} \cdot \frac{\Gamma_{24}-\Gamma_{15}}{2}\end{aligned}$ \\
\hline $\begin{array}{l}\Delta H_{2}= \pm \frac{c \gamma_{z z} \omega_{p}}{2 \varepsilon_{t} \sqrt{\mu_{p} / \varepsilon_{p}}} \cdot \frac{\Gamma_{12}-\Gamma_{45}}{2} \\
+\left[\frac{\chi \omega_{p}}{2 \varepsilon_{p}} \mp \frac{c \omega_{p}}{2 n_{p}} \cdot\left(\gamma_{x x}+\gamma_{y y}\right)\right] \cdot \frac{\Gamma_{12}+\Gamma_{45}}{2}\end{array}$ & $\begin{array}{l}\Delta H_{2}= \pm \frac{\alpha^{2} \cdot c \gamma_{z z} \omega_{p}}{2 \varepsilon_{t} \sqrt{\mu_{p} / \varepsilon_{p}}} \cdot \frac{\Gamma_{12}-\Gamma_{45}}{2} \\
+\left[\frac{\chi \omega_{p}}{\left(1+\kappa^{2}\right) \varepsilon_{p}} \mp \frac{c \omega_{p}}{\left(1+\kappa^{2}\right) n_{p}} \cdot\left(\gamma_{x x}+\gamma_{y y}\right)\right] \cdot \frac{\Gamma_{12}+\Gamma_{45}}{2}\end{array}$ \\
\hline$\Gamma_{1}$ & $\frac{1+\kappa}{2} \cdot \Gamma_{1}+\frac{1-\kappa}{2} \cdot \Gamma_{35}$ \\
\hline$\Gamma_{2}$ & $\frac{1+\kappa}{2} \cdot \Gamma_{2}+\frac{1-\kappa}{2} \cdot \Gamma_{34}$ \\
\hline$\Gamma_{4}$ & $\frac{1+\kappa}{2} \cdot \Gamma_{4}+\frac{1-\kappa}{2} \cdot \Gamma_{23}$ \\
\hline$\Gamma_{5}$ & $\frac{1+\kappa}{2} \cdot \Gamma_{5}+\frac{1-\kappa}{2} \cdot \Gamma_{13}$ \\
\hline
\end{tabular}

In order to obtain a Hamiltonian with only a few non-zero $a_{s}$ terms, only one or two nonzero terms are considered in our discussion, in Eq. (S8) when $\omega_{0} \neq 0$, we can modify the electromagnetic duality 
$\varepsilon_{t 0} \mu_{p} / \varepsilon_{p} \mu_{t 0}$ in the Drude model from 1 to a particular value to eliminate the difference of dispersion slope induced by the Lorenz term, that is $\kappa=\omega_{p} / \omega_{0} \cdot \sqrt{\varepsilon_{t 0} \mu_{p} / \varepsilon_{p} \mu_{t 0}}=1$. Therefore, $\kappa$ can be regarded as a quantitative measure of the effective electromagnetic duality in the Lorentz model. When the duality is satisfied, only a scale factor $\alpha$ is introduced to $v_{p}$ and those perturbation terms by the Lorentz resonance. It is worth mentioning that with a nonperfect balance parameter $\kappa \neq 1$, the modification from $\Gamma_{i}$ to $\frac{1+\kappa}{2} \cdot \Gamma_{i} \pm \frac{1-\kappa}{2} \cdot \Gamma_{3,6-i}$ introduces an additional $\Gamma_{3,6-i}$ term. Unlike the case with paired perturbed Hamiltonian, i.e., $\Delta H_{2}=a \cdot \Gamma_{12}+b \cdot \Gamma_{45}$, as will be shown in section I.E, this additional term will introduce some $k$-dependent perturbed Hamiltonians, which is equivalent to rotating the 5D Weyl surface system by a small angle. Consequently, the Weyl surfaces will be rotated from a pure $f\left(k_{i}\right)=0$ surface to a hybridized $f\left(k_{i}+\frac{1-\kappa}{2} \cdot k_{j}\right)=0$ surface. This rotation process behaves as the introduction of an effective mass term $m=\frac{1-\kappa}{2} \cdot k_{j}$ to the original degeneracy point. With judiciously engineered electric \& magnetic resonances and chiral \& bi-anisotropic properties, in the effective medium frame, the same perfect KPA Hamiltonian as Eq. (S8) can be obtained in a similar way as the case without the Lorenz resonance.

\section{Discussion on the importance of the antisymmetric bi-anisotropy matrix}

To have a Hamiltonian with only one or two non-zero $a_{s}$ terms in Eq. (S8), one needs to engineer the bi-anisotropic terms to satisfy the conditions: $\gamma_{x z}=-\gamma_{z x}$ and $\gamma_{y z}=-\gamma_{z y}$. These anti-symmetric bi-anisotropic terms can be realized through properly arranged split ring resonates. In the main text, we set these four bianisotropic terms to zero, which projects the 5D system to a particular 3D subspace ---the 3D laboratory momentum space.

On the other hand, when the bianisotropic terms do not come in anti-symmetric pairs, the Hamiltonian will become more complicated. Here, we discuss a particular case, with only a single bianisotropic term, i.e., $\gamma_{x z}$ without its antisymmetric counterpart, i.e., $\gamma_{z x}$. In this case, the effective Hamiltonian possesses both $\Phi_{4} \Gamma_{4}$ and $\Delta H_{3}=\Phi_{4} \Gamma_{23}$, and the Hamiltonian is $\boldsymbol{H}=\Phi_{3} I+\sum_{i=1}^{3} \Phi_{i} \boldsymbol{\Gamma}_{i}+$ $\Phi_{4} \boldsymbol{\Gamma}_{4}+\Phi_{4} \boldsymbol{\Gamma}_{23}$. Here, the Hamiltonian is similar to that in the main text $\boldsymbol{H}=\Phi_{3} I+\sum_{i=1}^{5} \Phi_{i} \boldsymbol{\Gamma}_{\boldsymbol{i}}+a$. $\Gamma_{m n}$, but it possesses a $k$-dependent perturbation coefficient: $a=\Phi_{4}=v_{p} k_{4}$. The eigenfrequency of such a Hamiltonian is $\delta \omega=\Phi_{3} \pm\left\{\sum_{i=1}^{4}{\Phi_{i}}^{2}+{\Phi_{4}}^{2} \pm 2 \Phi_{4} \sqrt{{\Phi_{1}}^{2}+{\Phi_{4}}^{2}+{\Phi_{5}}^{2}}\right\}^{\frac{1}{2}}$, and the degeneracy points can be calculated in the same way as that in the main text: 


$$
\begin{aligned}
& \mathcal{M}_{1}: \Phi_{2}=\Phi_{3}=0 \& \Phi_{1}^{2}+\Phi_{4}^{2}+\Phi_{5}^{2}=\Phi_{4}^{2} \rightarrow \mathcal{M}_{1}: \Phi_{1}=\Phi_{2}=\Phi_{3}=\Phi_{5}=0 \\
& \mathcal{M}_{2}: \Phi_{1}=\Phi_{4}=\Phi_{5}=0 \rightarrow a=0 \rightarrow \text { A 4-fold degeneracy Yang monopole at } k_{i}=0
\end{aligned}
$$

However, the manifolds of the degeneracy points are not two linked Weyl surfaces, but a 4-fold degenerate Yang monopole and a straight line along $k_{4}$ crossing the Yang monopole.

D. An intuitive connection between antisymmetric bi-anisotropy tensor and synthetic $\underline{\text { momenta }}$

In the following, we show that an antisymmetric bi-anisotropy matrix $\gamma$ can provide synthetic dimensions not only in our specific system but more universally in general electromagnetic media. Starting from Maxwell's equations with a homogeneous medium, the cross product ' $\vec{k} \times$ ' can be expressed in the tensor form as an antisymmetric matrix $\varepsilon_{m n p} k_{p}\left(\varepsilon_{m n p}\right.$ is the Levi-Civita symbol), which shows close resemblance to the antisymmetric matrix $\gamma_{m n}$. With the help of the Pauli and GellMann matrices, for a medium with antisymmetric bi-anisotropy matrices i.e., $\boldsymbol{\gamma}=-\boldsymbol{\gamma}^{T}$, Maxwell's equations can be formulated in an elegant form:

$$
\begin{gathered}
{\left[\left(\omega \gamma_{x y} \sigma_{x}-k_{z} \sigma_{y}\right) \cdot \lambda_{2}-\left(\omega \gamma_{z x} \sigma_{x}-k_{y} \sigma_{y}\right) \cdot \lambda_{5}+\left(\omega \gamma_{x y} \sigma_{x}-k_{z} \sigma_{y}\right) \cdot \lambda_{7}\right] \cdot \Psi_{E H}} \\
=\omega \cdot \frac{1}{2}\left[\left(\sigma_{0}+\sigma_{z}\right) \cdot \varepsilon_{0} \hat{\varepsilon}+\left(\sigma_{0}-\sigma_{z}\right) \cdot \mu_{0} \widehat{\boldsymbol{\mu}}\right] \cdot \Psi_{E H}
\end{gathered}
$$

Here, $\left\{\lambda_{2}, \lambda_{5}, \lambda_{7}\right\}$ are the three antisymmetric Gell-Mann matrices and $\Psi_{E H}=[E, H]^{T}$. Therefore, $\varepsilon_{m n p} k_{p}$ and $\omega \gamma_{m n}$ are on an equal footing for a general medium with arbitrary $\boldsymbol{\varepsilon}$ and $\boldsymbol{\mu}$. Therefore, an effective medium platform can offer up to six orthogonal effective momenta (three $k$ vectors and three bianisotropic terms), which are sufficient for the four-band system we are investigating (only five momenta are needed).

\section{E. Discussion of multiple perturbation terms}

In general, the combinations $\left[\gamma_{x x}+\gamma_{y y}, \gamma_{z z}\right]$ and $\left[\delta_{\varepsilon}, \delta_{p}\right]$ will introduce various $\Gamma_{m n}$ terms and we need to adjust the ratio of the perturbation parameters to realize the required Weyl surface Hamiltonian $H=H_{Y}+a \cdot \Gamma_{m n}$. The Hamiltonian can be transformed into $H=H_{Y}+a \cdot \Gamma_{m n}+b \cdot \Gamma_{i j}$ $(b<a)$ with two perturbation terms, in which the terms $[i, j, m, n]$ are the four unsorted dimension components excluding the particular $3^{\text {rd }}$ dimension, i.e., $H=H_{Y}+a \cdot \Gamma_{45}+b \cdot \Gamma_{12}$ or $H=H_{Y}+a$. $\Gamma_{15}+b \cdot \Gamma_{24}$. The analytical dispersion of this Hamiltonian is quite complicated. Here, we only show the numerical result, as shown in Fig. S1. The result shows that the additional $b \cdot \Gamma_{i j}$ term only deforms the 
detailed shape of the dispersion, but without affecting the topology of the linked Weyl surfaces. It is worth mentioning that the measured metamaterial exhibiting Weyl surface with $\Delta H=a \cdot \Gamma_{15}$ in Fig. 2 in the main text possesses a small $b \cdot \Gamma_{24}$ perturbation, which breaks the overlap between the two nodal lines projected by $\mathcal{M}_{2}$, but it keeps the linking property protected by the $C_{2}$ topology.

In summary, the KPA-Hamiltonian is a good approximation that can describe the linked Weyl surface's main features in the effective medium model, where the antisymmetric bi-anisotropic items can serve as synthetic momenta.

\section{The Second $\underline{k \cdot p}$ effective Hamiltonian of the Weyl surfaces}

The 'Weyl' nature of any point located on the degeneracy surface can be verified through a second $k \cdot p$ perturbed process. We expand the effective Weyl surface Hamiltonian $H_{W S}=\omega_{k 0} \cdot I+$ $\sum_{i=1}^{5} \Phi_{i}(\vec{k}) \cdot \Gamma_{i}+a \cdot \Gamma_{m n}=H_{Y}+a \cdot \Gamma_{m n}$ in the vicinity of any one of two-fold degeneracy points $\vec{K}_{W S}=\vec{K}_{Y}+\vec{p}$, and solve its corresponding 2-by-2 Hamiltonian $H=\sum_{i=0}^{3} \xi_{i}(\vec{K}) \cdot \sigma_{i}$ on the point $\vec{K}=$ $\vec{K}_{W S}+\vec{K}_{R}$, with $\vec{K}_{R}=\left[k_{R i}, k_{R j}, k_{R 3}, k_{R m}, k_{R n}\right]$ and $\left|\vec{K}_{R}\right| \ll 1$.

For a typical two-fold degeneracy point on the $S^{2}$ ellipsoid Weyl surface $\mathcal{M}_{1}$ with $\vec{p}(\theta, \phi)=$ $\left[p_{i}, p_{j}, p_{3}, p_{m}, p_{n}\right]=a \cdot\left[v_{p}^{-1} \cdot \sin \theta \cos \phi, v_{p}^{-1} \cdot \sin \theta \sin \phi, v_{t}^{-1} \cdot \cos \theta, 0,0\right]$, the expansion coefficients are: $\quad \vec{\xi}_{\mathcal{M}_{1}}(\theta, \phi)=\left[\operatorname{acos} \theta ; v_{p} \cos \phi \cdot k_{R m}+v_{p} \sin \phi \cdot k_{R n},-v_{p} \sin \phi \cdot k_{R m}+v_{p} \cos \phi \cdot k_{R n}, \vec{v}_{\mathcal{M}_{1}} \cdot \vec{K}_{\mathcal{M}_{1}}\right]$ with $\vec{v}_{\mathcal{M}_{1}}=\left[v_{p}, v_{p}, v_{t}\right]$ and $\vec{K}_{\mathcal{M}_{1}}=\left[k_{R i} \cdot \sin \theta \cos \phi, k_{R j} \cdot \sin \theta \sin \phi, k_{R 3} \cdot \cos \theta\right]$. Here, $\vec{v}_{\mathcal{M}_{1}} \cdot \vec{K}_{\mathcal{M}_{1}}$ denotes the direction of the surface normal of the ellipsoid Weyl surface $\mathcal{M}_{1}$. Therefore, from the perspective of a complete 5D space, each degeneracy point on the $S^{2}$ ellipsoid $\mathcal{M}_{1}$ can be regarded as a Weyl point in its corresponding 3-codimensional subspace $\left[K_{\mathcal{M}_{1}}, k_{R m}, k_{R n}\right]$. The frequency range of this Weyl surface is from $\omega_{p}-a$ to $\omega_{p}+a$. In general, the corresponding topological invariant (chirality) of a Weyl point with effective Hamiltonian $H=\sum_{i j} k_{i} v_{i j} \sigma_{j}$ can be defined through the standard form $c=$ $\operatorname{sgn}\left[\operatorname{det}\left(v_{i j}\right)\right]$. For the pair of Weyl points on $[\theta, \phi]$ and $[\pi-\theta, \pi+\phi]$ with the same codimension subspace, the chirality is always opposite, with one +1 and one -1 . Similar results can be obtained for the Weyl surface near the Yang monopole located at $-k_{D}$.

For a degeneracy point located on the $T^{2}$ Weyl surface plane $\mathcal{M}_{2}$ with $\vec{p}=\left[0,0,0, p_{m}, p_{n}\right]$, the expansion coefficients are: $\vec{\xi}_{\mathcal{M}_{2}}\left(p_{m}, p_{n}\right)=\frac{1}{\omega_{\mathcal{M} 2}} \cdot\left[ \pm \omega_{\mathcal{M} 2}^{2} \pm v_{p}^{2} \cdot\left(p_{m} k_{R m}+p_{n} k_{R n}\right) ; \mp a \cdot v_{p} k_{R i}, \pm a \cdot\right.$ $\left.v_{p} k_{R j}, \pm a \cdot v_{t} k_{R 3}\right)$ with $\omega_{\mathcal{M} 2}=\sqrt{a^{2}+v_{p}^{2} \cdot\left(p_{m}^{2}+p_{n}^{2}\right)}$. Here, the ' \pm ' sign denotes the higher/lower frequency degeneracy $\mathcal{M}_{2}$ surface near the positive Yang monopole. Therefore, from the perspective of 
the full 5D space, each degeneracy points on the $T^{2}$ plane $\mathcal{M}_{2}$ can be regarded as a Weyl point in the fixed 3-codimensional subspace $\left[k_{R i}, k_{R j}, k_{R 3}\right]$. The Weyl surface $\mathcal{M}_{2}$ is located either below the frequency $\omega_{p}-a$ or above the frequency $\omega_{p}+a$. The corresponding topological invariants of such a pair of Weyl points are also opposite to each other. Near the Yang monopole located at $-k_{D}$, the only changed term is a negative $k_{R 3}$ and the topology invariant is also \pm 1 for any Weyl point pair.

Indeed, the choice of different $[m, n]$ only rotates the Weyl surfaces' orientation in the $5 \mathrm{D}$ space but does not affect the system's topology property. However, in the particular 3D laboratory subspace with $k_{4}=k_{5}=0$, the cross-sections of the Weyl surface will look very different for different choices of $[m, n]$. For example, the Weyl surface with $\Delta H=a \cdot \Gamma_{45}$ have $\vec{\xi}_{\mathcal{M}_{1}}(\theta, \phi)=\left[a \cdot \cos \theta ; 0,0, \vec{v}_{\mathcal{M}_{1}}\right.$. $\left.\vec{K}_{\mathcal{M}_{1}}\right]$ and $\vec{\xi}_{\mathcal{M}_{2}}=\left[ \pm a ; \mp v_{p} k_{R 1}, \pm v_{p} k_{R 2}, \pm v_{t} k_{R 3}\right]$, which construct two degeneracy Weyl points with opposite chirality wrapped by a $S^{2}$ nodal surface without a topological charge; while for the Weyl surface with $\Delta H=a \cdot \Gamma_{15}$, we have $\vec{\xi}_{\mathcal{M}_{1}}(\theta)=\left[a \cdot \cos \theta ; v_{p} k_{R 1}, 0, v_{p} k_{R 2} \sin \theta+v_{t} k_{R 3} \cos \theta\right]$ and $\vec{\xi}_{\mathcal{M}_{2}}=\frac{1}{\omega_{\mathcal{M} 2}} \cdot\left[ \pm \omega_{\mathcal{M} 2}^{2} \pm v_{p}^{2} \cdot p_{1} k_{R 1} ; 0, \pm a \cdot v_{p} k_{R 2}, \pm a \cdot v_{t} k_{R 3}\right] \quad$ with $\quad \omega_{\mathcal{M} 2}=\sqrt{a^{2}+v_{p}^{2} \cdot p_{1}^{2}}$, which behaves like two linked nodal lines.

\section{Second Chern number $\underline{C_{2}}$ and Linking number}

\section{A. Non-Abelian $2^{\text {nd }}$ Chern number $\mathrm{C}_{2}$ in Yang monopole}

For the Yang monopole system with Hamiltonian $H_{Y}=\omega_{k 0} \cdot I+\sum_{i=1}^{5} v_{i} k_{i} \cdot \Gamma_{i}$, the band structures are fourfold degenerate at $\vec{k}=0$ with linear dispersion and twofold degenerate everywhere else. To define its Berry connection, the twofold degeneracy requires a $U(2)$ berry connection, which is defined as: $A_{i}^{\alpha \beta}(\vec{k})=i \cdot\left\langle u_{K}^{\alpha}\left|\partial_{k_{i}}\right| u_{K}^{\beta}\right\rangle$, with $\left|u_{K}^{\alpha}\right\rangle(\alpha=1,2)$ the two degeneracy eigenmodes of the valence bands and the Non-Abelian $U(2)$ Berry curvature can be solved directly: $F_{i j}=\partial_{k_{i}} A_{j}-\partial_{k_{j}} A_{i}-i$. $\left[A_{i}, A_{j}\right]$. Along the 4D sphere enclosing the fourfold degeneracy point $\vec{k}=0$, the Non-Abelian Chern number $C_{2}^{N A}$ can be directly solved:

$$
C_{2}^{N A}=\frac{1}{32 \pi^{2}} \cdot \oint_{S_{4}} d k^{4} \cdot \epsilon^{i j k l}\left[\operatorname{tr}\left(F_{i j} F_{k l}\right)-\operatorname{tr}\left(F_{i j}\right) \cdot \operatorname{tr}\left(F_{k l}\right)\right]= \pm 1
$$

The nonzero $C_{2}^{N A}$ implies the point at $\vec{k}=0$ is a Yang monopole, which is the source of the $S U(2)$ gauge field in 5D space. 


\section{B. Abelian $2^{\text {nd }}$ Chern number $\mathrm{C}_{2}$ in Weyl surface}

In the Weyl surface system with Hamiltonian $H=H_{Y}+a \cdot \Gamma_{m n}$, the $\boldsymbol{T P}$ breaking perturbation term $a \cdot \Gamma_{m n}$ deforms the original fourfold degeneracy Yang monopole to two two-fold degeneracy Weyl surfaces, and the initial two-fold degenerate dispersion is lifted. An Abelian $U(1)$ Berry curvature is defined in this Weyl surface system: $A_{i}^{\alpha}(\vec{k})=i \cdot\left\langle u_{K}^{\alpha}\left|\partial_{k_{i}}\right| u_{K}^{\alpha}\right\rangle$, with $\left|u_{K}^{\alpha}\right\rangle$ the $\alpha$ th eigenmodes. The Abelian U(1) Berry curvature can be solved directly: $F_{i j}=\partial_{k_{i}} A_{j}-\partial_{k_{j}} A_{i}$. One can define the $U(1)$ second Chern number of this Weyl surface $\mathcal{M}_{j}$ for band $\alpha$ on a 4D closed manifold $\mathcal{V}$ that encloses only $\mathcal{M}_{j}$ :

$$
C_{2}^{A}\left(\mathcal{M}_{j}, \alpha\right)=\frac{1}{32 \pi^{2}} \cdot \oint_{\mathcal{V}} d k^{4} \cdot \epsilon^{i j k l} F_{i j} F_{k l}= \pm 1
$$

It is worth noting that a typical $4 \mathrm{D}$ closed $\mathcal{V}$ is a narrow torus, which is shown as the gray torus in Fig. 1C in the main text. It can be considered as the direct product of the Weyl surface $\mathcal{M}_{j}$ and a $S^{2}$ surface which encloses a degeneracy Weyl point on $\mathcal{M}_{j}$.

Clearly, $C_{2}^{N A}=C_{2}^{A}= \pm 1$. This result is consistent with the perturbation process from the Yang monopole to the Weyl surface.

\section{Linking between $2^{\text {nd }}$ Chern Number $\mathrm{C}_{2}$ and the linking number}

This part closely follows Ref. (23-25) and will give a brief description of the connection between the second Chern number $C_{2}$ and the linking number between different Weyl surfaces. Specially, one can use $\mathcal{M}_{n, n+1}$ to denote the Weyl surface between the $n$-th and (n+1)-th band and here we assume only one Weyl surface between two adjacent bands. Similar to the Weyl point in 3D, Weyl Surface in 5D can also be viewed as a "monopole" of Berry connection, so:

$$
d F_{n}=2 \pi \cdot\left[\int_{k^{\prime} \in \mathcal{M}_{n-1, n}} \star \delta_{5}\left(k-k^{\prime}\right)-\int_{k^{\prime} \in \mathcal{M}_{n, n+1}} \star \delta_{5}\left(k-k^{\prime}\right)\right]=d\left[F_{n}^{(n-1, n)}-F_{n}^{(n, n+1)}\right](S
$$

The operator $\star$ denotes Hodge dual and the positive/negative sign specifies the positive orientation of each connected Weyl surface. Here, $F_{n}$ is defined by the $n$-th eigenstate, with $A=A_{\mu}(k) d k^{\mu}=$ $i\left\langle u_{k}^{n}\left|\partial_{k_{\mu}}\right| u_{k}^{n}\right\rangle d k^{\mu}, F=d A=F_{\mu \nu} d k^{\mu} \wedge d k^{v}$ and $F_{\mu \nu}=\partial_{[\mu} A_{\nu]}=\frac{1}{2}\left(\partial_{\mu} A_{\nu}-\partial_{\nu} A_{\mu}\right)$. The singularities of the Berry curvature come from two sets of monopoles between the $[n-1, n]$ bands and the $[n, n+1]$ bands, respectively. 
Define a special integral region $\partial V$ to calculate $C_{2}$, which only contains $\mathcal{M}_{n, n+1}$ and has a 4D "narrow tube" boundary which is topologically equal to $\partial V=\mathcal{M}_{n, n+1} \times S^{2}$, with $V=\mathcal{M}_{n, n+1} \times V_{S}$, and $\partial V_{S}=S^{2}$. So, integrating $F_{n}$ defined by the $n$-th eigenstate and using Stokes' theorem, we have:

$$
\begin{aligned}
& \left(\boldsymbol{C}_{2}\right)_{n}^{\partial \boldsymbol{V}} \equiv \oint_{\partial \boldsymbol{V}} \frac{1}{2}\left(\frac{\boldsymbol{F}_{n}}{2 \pi}\right)^{2}=\int_{\boldsymbol{V}} \frac{\boldsymbol{d} \boldsymbol{F}_{n}}{2 \pi} \frac{\boldsymbol{F}_{n}}{2 \pi}=\int_{\boldsymbol{V}}\left(\int_{\boldsymbol{k}^{\prime} \in \mathcal{M}_{n-1, n}} \star \boldsymbol{\delta}_{5}\left(\boldsymbol{k}-\boldsymbol{k}^{\prime}\right)-\int_{\boldsymbol{k}^{\prime} \in \mathcal{M}_{n, n+1}} \star \boldsymbol{\delta}_{5}\left(\boldsymbol{k}-\boldsymbol{k}^{\prime}\right)\right) \frac{\boldsymbol{F}_{n}}{2 \pi} \\
& \stackrel{\partial \boldsymbol{V}=\mathcal{M}_{n, n+1} \times \boldsymbol{S}^{2}}{\longrightarrow}\left(\boldsymbol{C}_{2}\right)_{n}^{\partial \boldsymbol{V}}=-\oint_{\mathcal{M}_{n, n+1}} \frac{\boldsymbol{F}_{\boldsymbol{n}}}{2 \pi}
\end{aligned}
$$

Here, $F_{n}$ consists of two parts. As verified in ref. $(23), F_{n}^{(n-1, n)}$ is not singular, and the integral of $F_{n}^{(n, n+1)}$ is zero. Using Stokes' theorem again, we have:

$$
\left(\boldsymbol{C}_{2}\right)_{n}^{\partial \boldsymbol{V}}=-\oint_{\mathcal{M}_{n, n+1}} \frac{\boldsymbol{F}_{n}{ }^{(\boldsymbol{n}-1, \boldsymbol{n})}}{2 \pi}=-\int_{\Sigma_{n, n+1}} \frac{\boldsymbol{d F}_{\boldsymbol{n}}^{(\boldsymbol{n}-1, \boldsymbol{n})}}{2 \pi}=-\int_{\boldsymbol{k} \in \Sigma_{n, n+1}} \int_{\boldsymbol{k}^{\prime} \in \mathcal{M}_{n-1, n}} \star \boldsymbol{\delta}_{5}\left(\boldsymbol{k}-\boldsymbol{k}^{\prime}\right)=-\mathcal{L}_{\mathcal{M}_{n, n+1}, \mathcal{M}_{n-1, n}}
$$

with $\partial \sum_{n, n+1}=\mathcal{M}_{n, n+1}$. The last term $\mathcal{L}_{\boldsymbol{W}_{n, n+1}, \boldsymbol{W}_{n-1, n}}$ counts the (signed) number of the intersection points. This term describes the intersection point of $\sum_{n, n+1}$ and $\mathcal{M}_{n-1, n}$, which is equivalent to the linking number of two 2-manifolds $\partial \sum_{n, n+1}=\mathcal{M}_{n, n+1}$ and $\mathcal{M}_{n-1, n}$.

Besides, by distinguishing the nonzero part of $F_{n}$ in the specific integration process, $C_{2}$ can be divided into two individuals $C_{1}$ number:

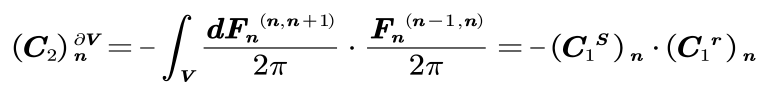

with the definitions: $\left(\boldsymbol{C}_{1}{ }^{\boldsymbol{S}}\right)_{n}=\int_{\boldsymbol{V}_{\boldsymbol{s}}} \int_{\boldsymbol{k}^{\prime} \in \boldsymbol{W}_{n, n+1}} \star \boldsymbol{\delta}_{5}\left(\boldsymbol{k}-\boldsymbol{k}^{\prime}\right)=\oint_{S^{2}} \frac{\boldsymbol{F}_{n}^{(n, n+1)}}{2 \pi} \&\left(\boldsymbol{C}_{1}{ }^{r}\right)_{n}=\oint_{\boldsymbol{W}_{n, n+1}} \frac{\boldsymbol{F}_{n}{ }^{(\boldsymbol{n}-1, n)}}{2 \pi}$.

Note that $C_{1}^{S}$ always equals to \pm 1 , which simply implies the fact that a Weyl surface can be viewed as a Weyl node in the three codimensions orthogonal to the Weyl surface, and $C_{1}^{r}$ describes the topology of the Weyl surface itself. It can be verified $\left(C_{2}\right)_{n}^{\partial V}=-\left(C_{2}\right)_{n+1}^{\partial V}$. The subscript ' $n+1$ ' represents the Berry connection defined by the $(n+1)-t h$ eigenstate. Through a proper choice of the orientation of $S^{2}$, we always have: $\left(C_{1}^{s}\right)_{n}=\left(C_{1}^{s}\right)_{n+1}$ and $\left(C_{1}^{r}\right)_{n}=-\left(C_{1}^{r}\right)_{n+1}$. These terms are responsible for the 1D Weyl Arc on the 3D Fermi Arc hypersurface.

\section{Real metamaterial design for the Weyl surface system}

\section{A. Design of structures based on the group symmetry}

Neumann's principle: if a crystal is invariant with respect to certain symmetry operations, any of its physical properties must also be invariant with respect to the same symmetry operations.

The Neumann's principle states that crystal symmetry exerts some influence on the symmetry of the physical properties. Specifically, considering a symmetry operator $g$ which transforms the crystal from 
an orthogonal $\left[x_{1}, x_{2}, x_{3}\right]$ system to another similarly orthogonal $\left[x_{1}^{\prime}, x_{2}^{\prime}, x_{3}^{\prime}\right]$ system with $x_{i}^{\prime}=A_{\alpha i} x_{\alpha}$ and $A_{\alpha i}=[A(g)]_{\alpha i}$, for a rank-2 polar tensor $\boldsymbol{d}$, e.g., $\boldsymbol{\varepsilon}, \boldsymbol{\mu}$, according to the definition, we have: $d_{i j}{ }^{\prime}=$ $A_{\alpha i} A_{\beta j} d_{\alpha \beta}$, while for a rank-2 pseudo tensor $\boldsymbol{d}$, i.e., $\boldsymbol{\gamma}$, we have: $d_{i j}{ }^{\prime}=\|R\| \cdot \operatorname{det}[A(g)] \cdot A_{\alpha i} A_{\beta j} d_{\alpha \beta}$, with ||$R||= \pm 1$ for the operator with/without a time reversal symmetry. Then the condition $d_{i j}^{\prime}=d_{i j}$ from Neumann principle will put a constraint on the form of tensor $\boldsymbol{d}$.

With a point Group symmetry $C_{4 h}$, which includes the rotation symmetry operator $C_{4}(z)$ and the mirror symmetry operator $\sigma_{z}$, the above medium tensors must have the forms:

$$
\boldsymbol{\varepsilon}=\operatorname{diag}\left(\varepsilon_{p}, \varepsilon_{p}, \varepsilon_{t}\right), \boldsymbol{\mu}=\operatorname{diag}\left(\mu_{p}, \mu_{p}, \mu_{t}\right) \text { and } \boldsymbol{\gamma}=0
$$

Hence a metamaterial geometry with a point group $C_{4 h}$ can be used to realize the 3D Dirac point, as shown in Fig. S2A. This 3D Dirac points can be viewed as one intersection $k_{4}=k_{5}=0$ of the Yang monopoles by considering the bianisotropic terms as the two additional dimensions.

To realize the linked Weyl surfaces with $\Delta H=a \cdot \Gamma_{45}$, with $\gamma_{z z}=\frac{2 \cdot \varepsilon_{t} / \varepsilon_{p}}{\alpha^{2} \cdot\left(1+\kappa^{2}\right)} \cdot\left(\gamma_{x x}+\gamma_{y y}\right)=$ $C_{c r i t i c a l} \cdot\left(\gamma_{x x}+\gamma_{y y}\right)$, we need to preserve the forms of the two rank-2 polar tensors $\boldsymbol{\varepsilon}$, $\boldsymbol{\mu}$, and meanwhile introduce a nonzero $\boldsymbol{\gamma}$ matrix with the formula $\boldsymbol{\gamma}=\operatorname{diag}\left(\gamma_{x x}, \gamma_{y y}, \gamma_{z z}\right)$, in which we fix $k_{4}=k_{5}=0$. A perturbation structure satisfying $D_{4}$ point symmetry is used, which contains the rotation symmetry operator $C_{4}(z), C_{2}(x), C_{2}(y)$ and leads to the following tensor forms:

$$
\boldsymbol{\varepsilon}=\operatorname{diag}\left(\varepsilon_{p}, \varepsilon_{p}, \varepsilon_{t}\right), \boldsymbol{\mu}=\operatorname{diag}\left(\mu_{p}, \mu_{p}, \mu_{t}\right) \text { and } \boldsymbol{\gamma}=\operatorname{diag}\left(\gamma_{p}, \gamma_{p}, \gamma_{t}\right)
$$

It should be noted that the $C_{4}$ point symmetry of the final structure allows a nonzero $\varepsilon_{x y}$ or $\mu_{x y}$ term. However, these additional terms can be tuned to zero with a semicircular metallic helix perturbation unit, as shown in Fig. S2B-C.

Similarly, to realize the linked Weyl surface with $\Delta H=a \cdot \Gamma_{15}$, with $\delta_{\varepsilon}=4 \varepsilon_{p} \delta_{p}$, we introduce an additional metallic bar to realize $\delta_{\varepsilon}$ and a thinner dielectric spacer layer to break the degeneracy between the electric and the magnetic longitude modes, as shown in Fig. S2D-E. This structure reduces the $C_{4}(z)$ axis in the original Dirac point structure into a $C_{2}(z)$ axis, then the full unit satisfies the $C_{2 h}$ point symmetry. The parameters must be in the following forms:

$$
\boldsymbol{\varepsilon}=\operatorname{diag}\left(\varepsilon_{x x}, \varepsilon_{y y}, \varepsilon_{z z}\right), \boldsymbol{\mu}=\operatorname{diag}\left(\mu_{x x}, \mu_{y y}, \mu_{z z}\right) \text { and } \boldsymbol{\gamma}=0
$$

In our design, we only introduce an additional electric response, so we will still have $\mu_{x x}=\mu_{y y}$.

The details of the structure geometry are listed below: in Fig. S3A: $r=1.2 \mathrm{~mm}, w=0.2 \mathrm{~mm}, d=$ $1.0 \mathrm{~mm}, g=0.6 \mathrm{~mm}, d_{I}=3.0 \mathrm{~mm}, p_{x}=p_{y}=6.8 \mathrm{~mm}$; in Fig. S3B $: r_{I}=1.2 \mathrm{~mm}, d_{L I}=0.5 \mathrm{~mm}, d_{I}^{45}=$ 
3.0mm; and in Fig. S3C: $L_{I}=6.5 \mathrm{~mm}, w_{I}=0.2 \mathrm{~mm}, d_{I}^{15}=1.0 \mathrm{~mm}$. Besides, the permittivity of the dielectric layers is 1.96 [F4BM220] in the simulation, and metal is treated as PEC.

The dispersions of these designed structures are calculated by the eigenmode solver of CST Studio Suite, and the detailed results are shown in Fig. S4. With increasing $k_{x}$ or $k_{y}$, the degeneracy points will open and exhibit anti-crossing features. Compare the results with that in Fig. 1B in the main text, the effective Hamiltonian model of these structures can be verified.

\section{B. Effective medium coefficients of the designed metamaterials}

To better understand the system we designed, we fit the dispersion data based on EMT model and obtain the corresponding KPA Hamiltonian coefficients for the designed structures above. This KPA Hamiltonian model can describe the dispersion results well for both zero and finite $k_{x}\left(k_{y}\right)$ cases.

For the Dirac point system, as shown in Fig. S2A, the effective medium model satisfies Eq. (S22). To fit the simulation results, we introduce two additional velocity scaling factors $g_{p}$ and $g_{t}$, which result in modified Fermi velocity $v_{p}=g_{p} \cdot v_{p, K P A}=g_{p} \cdot \frac{c}{2 \sqrt{\varepsilon_{t} \mu_{p}}}$ and $v_{t}=g_{t} \cdot v_{t, K P A}=g_{t} \cdot \frac{c}{2 \sqrt{\varepsilon_{p} \mu_{p}}}$ in the KPA Hamiltonian. The fitting parameters are $\varepsilon_{p}=3.03, \varepsilon_{t 0}=\mu_{p}=\mu_{t 0}=1.0, \omega_{p}=$ $7.95 \mathrm{GHz}, \omega_{0}=7.2 \mathrm{GHz}, g_{p}=0.6$ and $g_{t}=0.8$, and the effective Hamiltonian satisfies $H_{Y}=\omega_{k 0}$. $I+\sum_{i=1}^{5} v_{i} k_{i} \cdot \Gamma_{i}$ with $\kappa=0.634$. The dispersion results calculated by the effective Hamiltonian perfectly match the original simulation results, as shown in Fig. S5A-B. However, the offset of $\kappa$ from 1 affects the experimental detection of the Weyl arc, as shown in Fig. 3E-F in the main text. A detailed discussion about this part will be shown in section V.B.

For the Weyl surface structure designed in Fig. S2C, its effective medium model satisfies Eq. (S23). The fitting parameters are: $\varepsilon_{p}=\varepsilon_{t 0}=3.26, \mu_{p}=\mu_{t 0}=1.0, \omega_{p}=7.52 \mathrm{GHz}, \omega_{0}=6.92 \mathrm{GHz}$, $\gamma_{p} \omega_{p}=0.013 K_{D}, \gamma_{t} \omega_{p}=0.303 K_{D}, g_{p}=0.8$ and $g_{t}=0.77$. The effective Hamiltonian from these fitting parameters is $H_{W S}=H_{Y}+a \cdot \Gamma_{45}+b \cdot \Gamma_{12}$ with $a=-0.0179 \cdot \omega_{p}$ and $b=0.0057 \cdot \omega_{p}$, $(|a| \sim 3 \cdot|b|)$ and with an almost perfect effective electromagnetic duality $\kappa=1.09$. The dispersion results calculated by this KPA Hamiltonian perfectly match the simulation results, as shown in Fig. S5CD.

For the Weyl surface structure designed in Fig. S2E, the effective medium model satisfies: $\boldsymbol{\varepsilon}=$ $\operatorname{diag}\left(\varepsilon_{p}, \varepsilon_{p}+\delta_{\varepsilon}, \varepsilon_{t}\right), \boldsymbol{\mu}=\operatorname{diag}\left(\mu_{p}, \mu_{p}, \mu_{t}\right)$ and $\boldsymbol{\gamma}=0$, with $\omega_{p H, p E}=\omega_{p} \cdot\left(1 \pm \delta_{p}\right)$. The fitting parameters are: $\varepsilon_{p}=3.18, \varepsilon_{t 0}=2.2, \mu_{p}=\mu_{t 0}=1.0, \omega_{p}=7.92 \mathrm{GHz}, \omega_{0}=7.2 \mathrm{GHz}, \delta_{\varepsilon}=0.55$, 
$\delta_{p}=0.018, g_{p}=1.0$ and $g_{t}=0.7$. The effective Hamiltonian from these fitting parameters is $H_{W S}=H_{Y}+a \cdot \Gamma_{15}+b \cdot \Gamma_{24}$ with $a=-0.0333 \cdot \omega_{p}$ and $b=0.0137 \cdot \omega_{p}(|a| \sim 2.5 \cdot|b|)$ and with $\kappa=0.92$. As shown in Fig. S5E-H, the dispersion results calculated by this effective Hamiltonian match well with the original simulation results with both zero/nonzero $k_{x}$ and $k_{y}$. It is worth noting that there are some other resonant modes near $6.5 \mathrm{GHz}$ in the designed structure that cannot be fully described by the 4-band KPA Hamiltonian model. These additional modes induce a small gap at the expected degenerate points projected by the 5D Weyl surface, as shown in Fig. S4. A detailed discussion on this part will be shown in section IV.D.

While the nominal dielectric constant of the dielectric media is 1.96 [F4BM220] in the CST simulation, the actual parameters for the permittivity can be a different value. In addition, both the original and the perturbed structures have some additional resonant modes, which slightly affect the response on the designed frequency. This leads to the different fitting permittivity values and the additional velocity scaling factors $g_{p}$ and $g_{t}$. Fortunately, this effect does not change the topology property of the systems. Besides, the effective fitting Hamiltonian always has two perturbation terms with the two coefficients satisfying $a \gg b$. Nonetheless, the presence of $b$ coefficient also does not influence the topology property of the system, as discussed in section I.E. In summary, the designed structures represent good candidates to study the Weyl surface system in a photonic metamaterial.

\section{Discussion about the gap in $a \cdot \Gamma_{15}$ perturbed system}

As shown in Fig. S4C-D, a small gap occurs near the expected degeneracy points in the Weyl surface system with $\Delta H=a \cdot \Gamma_{15}$, due to the presence of resonant modes near $6.5 \mathrm{GHz}$. The influence of these additional resonances can be described by a small equivalent mass term $m$. To quantitatively discuss this effect, we fit the simulated EFS data, as shown in Fig. S6.

The EFS of such a system without additional modes can be either elliptical or hyperbolic depending on the frequency region, which satisfies $\frac{k_{z, 0}^{2}}{a^{2}} \pm \frac{k_{\|, 0}^{2}}{b^{2}}=1$. To take into account the influence of an equivalent mass item $m$, the relationship of $k_{\|}-k_{z}$ can be solved as the eigenvalues of a coupling matrix, which is:

$$
\left[\begin{array}{cc}
k_{z, 0}^{(1)}\left(k_{\|}\right) & m \\
m & k_{z, 0}^{(2)}\left(k_{\|}\right)
\end{array}\right]=D \cdot\left[\begin{array}{cc}
k_{z}^{(1)}\left(k_{\|}\right) & 0 \\
0 & k_{z}^{(2)}\left(k_{\|}\right)
\end{array}\right] \cdot D^{-1}
$$


From the simulated EFS data, we can fit the original EFS and the strength of the effective mass term. As shown in Fig. S6, the fitted data exactly match the simulated EFS data at all frequencies. Besides, the effective influence $m$ is more pronounced in the relatively low-frequency region, as shown in Fig. S6H, which can prove that the influence mainly comes from additional resonances near $6.5 \mathrm{GHz}$.

\section{Fermi Hypersurface and Weyl Arc}

\section{A. The existence of Weyl Arc in systems possessing nonzero $\mathrm{C}_{2}$}

For the Yang monopole system, any cross-section with fixed $k_{z}$ located between (outside) the two Yang Monopoles constructs a $4 \mathrm{D}$ gapped system with $\left|C_{2}\right|=1(0)$. Such a system with nontrivial $C_{2}$ is known to exhibit the 4D quantum Hall effect and host surface states with surface Weyl points $(14,15)$. By gradually shifting $k_{z}$ between two Yang monopoles of opposite $C_{2}$, the Weyl points form a 1D arc, i.e., Weyl arc, that extends from one Yang monopole to the other, as shown in Fig. 3C-D.

The existence of Weyl arc in a system with Yang monopole or linked Weyl surfaces can also be explained by decomposing the second Chern number into the product of two first Chern numbers, i.e., $C_{2}^{A}=-C_{1}^{r} C_{1}^{s}$, as shown in section III.C. In this framework, we can make a correspondence between a $S^{2}$ manifold sub-EFS of the Yang monopole to the non-trivial Weyl surfaces with $\left|C_{1}^{r}\right|=1$. As shown in Fig. S7A-B, the 3-manifold Fermi arc hypersurface, with 2D cross-section homotopic to the nontrivial EFS close to the Yang monopole frequency, cannot contract to zero by itself and must connect to the other EFS with the same $C_{1}^{r}$. For $\delta \omega>0$, this connection is protected by topology, and only slightly affected by the EFS of air (the gray cylinder in the middle). When the frequency decreases below the degeneracy point $(\delta \omega<0)$, the sign of $C_{2}$ is reversed (the same $C_{1}^{s}$ but opposite $C_{1}^{r}$ ), and $C_{1}^{r}$ of the $2 \mathrm{D}$ cross-section of the Fermi arc hypersurface close to the EFS also changes sign. However, $C_{1}^{r}$ of the cross-section of the hypersurface in the middle of the connection still preserves its sign. As a result, Weyl nodes appear on the 3-manifold hypersurface between 2D cross-sections with opposite $C_{1}^{r}$. These nodes emerge from EFSs on both sides and approach each other as the frequency decreases.

As a comparison, for a system with Weyl surfaces, the results are similar to that of the Yang monopole, considering that Yang monopole is a limit of Weyl surface with $a=0$, except for the lifted degeneracy of bulk states, as shown in Fig. S7C. This Weyl arc described above for both the Yang monopole and Weyl surfaces is a unique phenomenon arising from the nontrivial second Chern number $C_{2}$. 


\section{B. The robustness of Weyl Arc (Numerical Analysis)}

As discussed above, the Weyl arc on the 3D Fermi hypersurface is protected by topology and must exist at the 4D boundaries. However, in our experiment, at the expected Weyl Arc point location, there is a small anti-crossing gap between different Fermi Arcs. This gap originates mainly from the impedance mismatch with the surrounding medium - air, as in the designed metamaterial, the electromagnetic duality is not satisfied. Despite this mismatch of impedance, we will show that the existence of the Weyl arc is still guaranteed at the $4 \mathrm{D}$ boundary.

For the realistic configuration of Yang monopole, the fitted medium parameters have a non-perfect electromagnetic duality with $\kappa=0.634$, which will introduce some other $\Gamma_{i j}$ terms with $k$-dependent coefficients, as discussed in section I.B. As a result, as shown in Fig. S8A-B, the 2-fold degeneracy of EFS is broken. Besides, this nonperfect condition will also destroy the impedance matching condition at the metamaterial/air boundary. Consequently, a small gap appears at the position of the expected Weyl arc point (blue circles). However, as discussed in section III, this violation of electromagnetic duality does not change the $2^{\text {nd }}$ Chen number $C_{2}$. Therefore, the existence of the Weyl arc is still guaranteed.

Indeed, we can modify the properties of either material across the metamaterial/air interface to satisfy the impedance match condition and render the gap closed in the 3D K-space. This condition, through some derivations, can be given as:

$$
\left.\frac{\varepsilon_{p} \varepsilon_{t}}{\mu_{p} \mu_{t}}\right|_{\text {air }}=\left.\frac{\varepsilon_{p} \varepsilon_{t}}{\mu_{p} \mu_{t}}\right|_{\text {metamaterial }}=\left.\frac{\omega_{p}^{2}}{\omega_{0}^{2}} \cdot \frac{\varepsilon_{p} \varepsilon_{t 0}}{\mu_{p} \mu_{t 0}}\right|_{\text {metamaterial }}
$$

Here, 'air' is replaced by a tunable uniaxial material. Clearly, an ideal design with $\kappa=1$ can satisfy the condition perfectly. As a comparison, the corresponding results of a modified medium: $\varepsilon_{p}=\varepsilon_{t}=\mu_{p 0}=$ $C, \mu_{t 0}=C \cdot \omega_{p}{ }^{2} / \omega_{0}{ }^{2}$, with $C=1.74$ a constant, are shown in Fig. S8C-D. Both the 2-fold degenerate EFS and the Weyl node/arc are observed in this modified system.

On the other hand, varying the synthetic $k_{4}$ and $k_{5}$ dimension can better prove the robustness of the Weyl arc arising from topological origin. Here, we add an arbitrary anti-symmetric $\gamma$ matrix to the fitted parameters and calculate the evolution of the gap at $k_{y}=0$, which is equivalent to varying the synthetic $k_{4}$ and $k_{5}$ dimensions. The results, as shown in Fig. S8E-G, verify that a perfect intersection of the Fermi arcs, which corresponds to a surface Weyl point, can always exist on different interfaces (a positive $k_{5}$ along the $\mathrm{x}$-direction and a negative $k_{4}$ along the $\mathrm{y}$-direction), and at different frequencies (from the Lorentz resonance frequency $7.2 \mathrm{GHz}$ to the bulk plasmon frequency $7.95 \mathrm{GHz}$ ). Besides, a 
numerical calculation shows that even without the impedance matching condition, the gap can close at the plasmonic frequency $\left(7.95 \mathrm{GHz}\right.$ in the calculation) without an additional $k_{4}$ or $k_{5}$ term, which has been observed in our experiments.

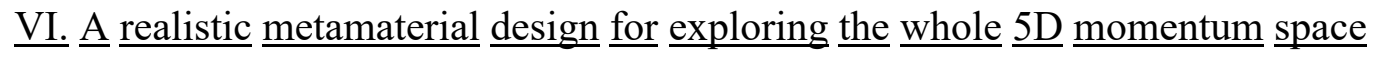

In this work we have focused on the most representative features (e.g., linking of Weyl surfaces and presence of Weyl arcs) of the nontrivial $C_{2}$ topology in 5D space. We have done this by rotating the Weyl surfaces in 5D space via changing $\Delta H$, so we can fix the subspace and avoid the difficulty of investigating the system in the synthetic dimensions $k_{4}$ and $k_{5}$. In this section, we will present the detailed real metamaterial designs for exploring the whole 5D momentum space, which are all backed by full-wave electromagnetic simulations.

\section{A. A realistic metamaterial with arbitrary synthetic momenta}

We first show the design of metamaterials to introduce $k_{4}$ and $k_{5}$ to the original Yang monopole configuration $(\Delta \mathrm{H}=0)$, as shown in Fig. S2A, in which the bianisotropic terms are completely canceled due to the presence of the inversion symmetry for the unit cell, which consists of four columns of helix pairs with mirror symmetry. Through the analysis based on the point group, the bianisotropic terms can be introduced by breaking the $C_{4}$ symmetry (and consequently the inversion symmetry). Specially, we can adjust the geometry and orientation of each column of helices to break the $C_{4}$ symmetry and thus to introduce the nonzero $k_{4}$ and $k_{5}$. The realistic design of the structure follows the guidance of circuitbased effective media analysis in Ref. (8), with the specific configurations shown in Fig. S9 A-D. For each individual helix, the key geometric parameter to be tuned is $\theta_{45}$ - the opening angle of split rings constituting the helix (Fig. S9B). This parameter controls the ratio between the symmetric (e.g., $\gamma_{x z}+$ $\gamma_{z x}$ ) and antisymmetric bianisotropic terms (e.g., $\gamma_{x z}-\gamma_{z x}$ ) for each column of helix pair (note that each column of helix pair alone exhibits bianisotropy because it does not have inversion symmetry). This angle is optimized to remove the symmetric term. When the four columns of helices are assembled together to form $C_{4}$ rotational symmetry, the bianisotropic terms from the four columns cancel each other, and this corresponds to the case of $k_{4}=k_{5}=0$ (Fig. S9C). Here the orientation angle $\psi_{45}$ of the helices controls the ratio between $k_{4}$ and $k_{5}$ once subsequent breaking of $C_{4}$ symmetry is introduced. The breaking of $C_{4}$ rotational symmetry is achieved by rotating the left two columns of helices in the clockwise direction by an angle $\delta_{45}$, and the right two columns in the counter-clockwise 
direction by the same angle $\delta_{45}$ (Fig. S9D), relative to the original $C_{4}$ configuration shown in Fig. S9C. Thus, the overall magnitude of synthetic momenta in the $k_{4}-k_{5}$ subspace, i.e., $\sqrt{k_{4}^{2}+k_{5}^{2}}$ is controlled by $\delta_{45}$.

Compared with the original $C_{4 h}$ structure with $k_{4}=k_{5}=0$, this configuration will not cause additional disturbance on $\boldsymbol{\varepsilon}$ and $\boldsymbol{\mu}$, and based on the circuit model, $\boldsymbol{\gamma}$ can be expressed as:

$$
\gamma \propto \frac{\omega r}{\omega^{2}-\omega_{0}^{2}+i \gamma_{0} \omega}\left(\begin{array}{ccc}
0 & 0 & 2 r^{2} \sin \theta_{45} \cdot\left(2 \pi-\theta_{45}\right) \cdot \cos \psi^{\prime} \\
0 & 0 & 2 r^{2} \sin \theta_{45} \cdot\left(2 \pi-\theta_{45}\right) \cdot \sin \psi^{\prime} \\
d^{2} \cdot\left(1-\cos \theta_{45}\right) \cdot \cos \psi^{\prime} & d^{2} \cdot\left(1-\cos \theta_{45}\right) \cdot \sin \psi^{\prime} & 0
\end{array}\right) \cdot \frac{\sqrt{2}}{2} \sin \delta_{45}
$$

where $\psi^{\prime}=\psi_{45}+\pi / 4$. Here, the radius of the split ring $r$ and the length of the pillar $d$ along $z$ direction dominate the magnetic and electric response, respectively. In the model, to realize a purely antisymmetric $\gamma$ response, the critical opening angle $\theta_{45}$ needs be carefully adjusted for a given combination of $r$ and $d$ to balance the magnitudes of $\left\{\gamma_{x z}, \gamma_{y z}\right\}$ and $\left\{\gamma_{z x}, \gamma_{z y}\right\}$, such that $d^{2}$. $\left(1-\cos \theta_{45}\right)+2 r^{2} \cdot \sin \theta_{45} \cdot\left(2 \pi-\theta_{45}\right)=0$. Thus, the magnitude and orientation of synthetic momenta in the $k_{4}-k_{5}$ subspace are controlled by $\delta_{45}$ and $\psi_{45}$, respectively.

The dispersion of such a realistic metamaterial calculated by full-wave simulation is shown in Fig. S9E. Along $k_{z}$ with $k_{x}=k_{y}=0$, the gap increases with continuously increasing $\delta_{45}$ for a fixed $\psi_{45}=\pi / 4$. This increased gap size indicates a continuously increased $k_{5}$, which can be numerically fitted by a sinusoidal function of the rotation angle $\delta_{45}$, as shown in Fig. S9F, confirming the prediction of the electrical circuit model. The slight lift of the degeneracy between $1^{\text {st }} \& 2^{\text {nd }}$ and $3^{\text {rd }} \& 4^{\text {th }}$ bands can be attributed to the fact that the designed structure slightly deviates from a purely antisymmetric gamma matrix, due to some higher-order effects. This results in a small residue $\Gamma_{13}$ term arising from the symmetrical part of $\gamma$ matrix, which is, nonetheless, only about $15 \%$ of $k_{5}$. Thus, this configuration represents a good candidate to realize a synthetic Yang monopole.

\section{B. The effect of the synthetic momenta on Weyl surfaces}

We further explore the cross-sections of the linked Weyl surfaces in the 3D real momentum subspace at different $k_{4}$ and $k_{5}$, as illustrated by Fig. S10A. We consider a similar configuration of Weyl surface metamaterial with $\Delta H=a \cdot \Gamma_{15}$ as shown in Fig. S2E. The thicknesses of the spacer layer $d_{I}$ is reduced to $1.0 \mathrm{~mm}$, and a metallic strip with section $6.5 \mathrm{~mm} \times 0.2 \mathrm{~mm}$ is inserted into the spacer layer. As we have discussed before, the synthetic momenta $k_{4}$ or $k_{5}$ are controlled by $\psi_{45}$ and $\delta_{45}$. 
We study the band evolution with increasing synthetic momentum $\left(k_{4}\right.$ or $k_{5}$ through continuously increasing $\delta_{45}$ ) for different combinations of $k_{x}$ and $k_{y}$, as shown in Figs. S9B-G. The degeneracy points belonging to the two Weyl surfaces are labeled by red $\left(M_{1}\right)$ and blue $\left(M_{2}\right)$ dots, respectively. The corresponding light-colored dots represent the slightly lifted degeneracy points affected by parasitic resonance terms, as discussed in section IV.C. Comparing with the results shown in Figs. S4C-D, these synthetic momenta behave in the same way as the real momenta $k_{x}$ and $k_{y}$. In the case of $\psi_{45}=\pi / 4$, an increased $\delta_{45}$ angle corresponds to an increased $k_{5}$, which lifts the $M_{1}$ nodal line degeneracy, and in the other case with $\psi_{45}=-\pi / 4$, an increasing $k_{4}$ shrinks the $M_{1}$ nodal line and lifts the $M_{2}$ nodal line degeneracy. This effect persists even when the system has a finite $k_{x}$ and $k_{y}$, and any real momentum cannot counteract the effects of these synthetic momenta because they are orthogonal in the 5D synthetic momentum space. A similar calculation is performed for a rotated metallic strip (by $90^{\circ}$ ) which corresponds to $\Delta H=a \cdot \Gamma_{24}$. Indeed, the configuration with a nonzero $k_{4}$ and $\Delta H=a \cdot \Gamma_{15}$ is exactly equivalent to the case with a nonzero $k_{5}$ and $\Delta H=a \cdot \Gamma_{24}$. This calculation verifies that the ratio between the synthetic momenta $k_{4}$ and $k_{5}$ can be finely controlled by $\psi_{45}$ and that the synthetic momenta can affect the Yang monopole/ Weyl surface system in the same way as the real momenta.

\section{Realistic metamaterials for precisely locating the surface Weyl points in synthetic space}

In section V.B, we show that the surface Weyl points in Yang monopole metamaterial, which are slightly shifted away from the $k_{z}$-axis due to the impedance mismatch, can be precisely located in the synthetic dimension. To verify this, we calculate the surface states confined to the boundary between a Yang monopole metamaterial (with tunable $k_{5}$ ) and air, as shown in Fig. S11. The Fermi Arcs at $k_{y}=$ 0 are calculated through the simulation of a Yang monopole sample consisting of six unit cells (shown in Fig. S9A) along the surface normal (x direction). A continuously increasing $\delta_{45}$ angle with $\psi_{45}=$ $\pi / 4$ is used to realize a variable synthetic momentum $k_{5} \cdot k_{5}$ can adjust the impedance properties of the surface and close the gap between two Fermi arcs, which is consistent with Fig. S8G. By continuously varying $k_{5}$, the surface Weyl points form an arc in the $k_{z}-k_{5}$ plane, whose frequency spans from the Lorentz resonance frequency to the bulk plasmon frequency, as illustrated in Fig. S11H. It should be noted that the gap between the two Fermi arcs near the Yang monopole frequency, as shown in Fig. S11A$\mathrm{B}$, arises from the coupling between the surface states on the opposite surfaces due to the finite number of unit cells and the very long decay length into the metamaterial. Thus, a finite-size calculation 
inevitably introduces the coupling between the surface states, which opens the gap and results in slight deviation from the prediction shown in Fig. S8B.

\section{Details of the Experiments}

\section{A. Experiment Setup}

Fig. S12B-C shows the schematic diagram of the experimental setups - two dipole antennas act as source (red) and probe (blue), respectively. The probe scans the top surface in a square grid to map out the projected states, with one or two points within each unit size. The direction of the probe is adjusted to match different modes. The source is buried inside the sample in the measurement of the linked Weyl surfaces to maximize the excitation of the bulk state, while it is placed near one edge of the top surface to maximize the excitation of the surface state for the measurement of the Weyl arcs. The inset of Fig. S12B-C shows the typical real space field distributions of the bulk/surface state measurements.

The samples, containing $20 \times 70 \times 70$ units, are constructed by stacking up the PCB layers. Each unit cell layer contains 4 PCB layers for the Yang monopole sample, and 5 PCB layers for the sample with linked Weyl surfaces, as shown in Fig. S12A. The lateral size of the PCB layer is $136 \mathrm{~mm} \times 476 \mathrm{~mm}(20 \times 70$ units). Through a standard Fourier transformation process, we can obtain the projection of the bulk/surface states on the effective $k_{x}-k_{z}$ or $k_{y}-k_{z}$ planes, as shown in Fig. 2 and Fig. 3 in the main text. In Fig. 2 in the main text, we rotate the direction of the layers containing metallic bars in different samples to measure both the $k_{y}-k_{z}$ and $k_{x}-k_{z}$ planes, as shown in Fig. S12 D.1 and Fig. S12 D.2.

\section{B. Detailed experimental results}

In Fig. S13, we compare the configurations of the bulk/surface states at different frequencies for the system with $\Delta H \approx a \cdot \Gamma_{15}$, obtained from EMT and the experimental measurements. The configuration of the Fermi arc surface states matches well with that of the experimental results. To detect bulk states more efficiently, the source is buried inside the sample to maximize the bulk state's excitation, as shown in Fig. S12B. However, the measured excitation still contains both the bulk states and the surface Fermi arc states. Indeed, the magnitude of the surface state is much stronger than the bulk state, even in this setup with a buried source. This leads to challenge in detecting the concrete intersection point of bulk state boundaries. 
In Fig. S14, we show detailed results corresponding to Fig. 3E in the main text. The results fit both EMT and CST simulation results well. The blue circles show the projection of the numerically calculated surface Weyl points in the 4D synthetic space of the boundary (see section V.B for details). In the 2D momentum subspace of the boundary, the finite synthetic $k_{4}-k_{5}$ parameters behave like an effective mass; therefore, a small anti-crossing gap appears near the position of the expected surface Weyl point. 


\section{Figures}

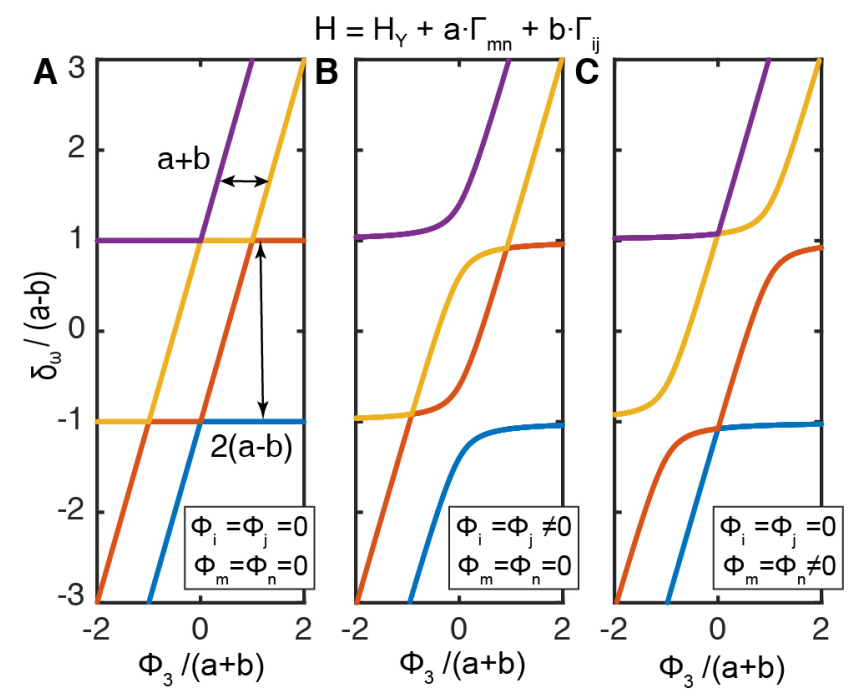

Fig. S1. Schematic dispersion of the Weyl surface in 5D space with two perturbation terms. (AC) shows the dispersion spectrum along $k_{3}$ for the general Weyl surface system $H=H_{Y}+a \cdot \Gamma_{m n}+$ $b \cdot \Gamma_{i j}$, for various $k_{m}\left(k_{n}\right)$ and $k_{i}\left(k_{j}\right)$ in different subgraphs. 


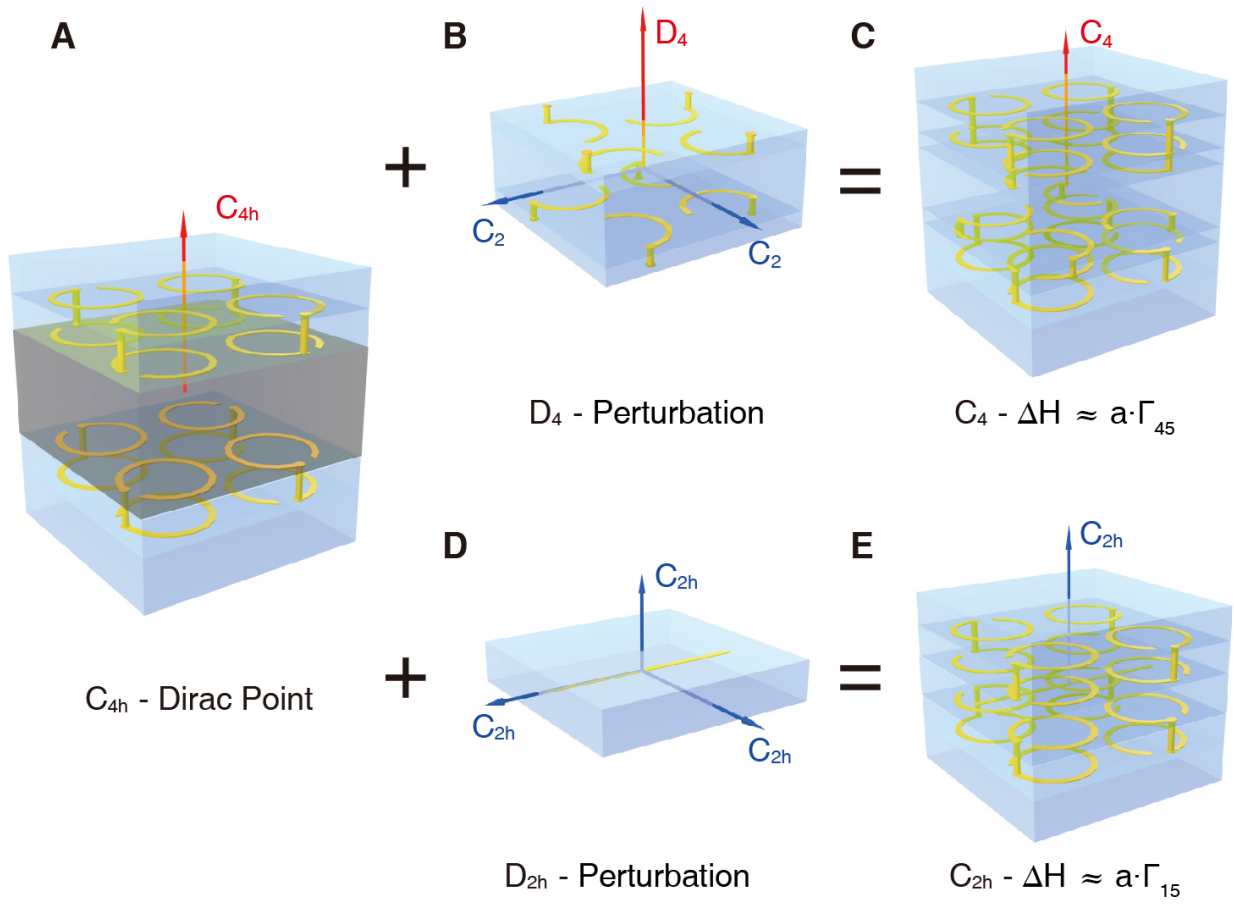

Fig. S2. Schematic of the designed Weyl surface metamaterials. (A) the configuration of the unit cell of a metamaterial exhibiting Dirac points, which consists of two layers of chiral metallic ring structures of opposite chirality. The overall unit cell exhibits $C_{4 h}$ symmetry. The Dirac points are promoted to Yang monopoles with two bianisotropic terms as additional synthetic dimensions. (B) Additional structure of $D_{4}$ symmetry, which, when added to the configuration in (A), leads to a new structure of $C_{4}$ symmetry, as shown in (C), which corresponds to a Weyl surface system with nonzero $\Delta H \approx a \cdot \Gamma_{45}$. (D, E) By introducing a thin metallic strip structure of symmetry $D_{2 h}$ (D) to the Yang monopole system shown in (A), a new configuration with $C_{2 h}$ symmetry is formed (E), which corresponds to a Weyl surface system with $\Delta H \approx a \cdot \Gamma_{15}$. 

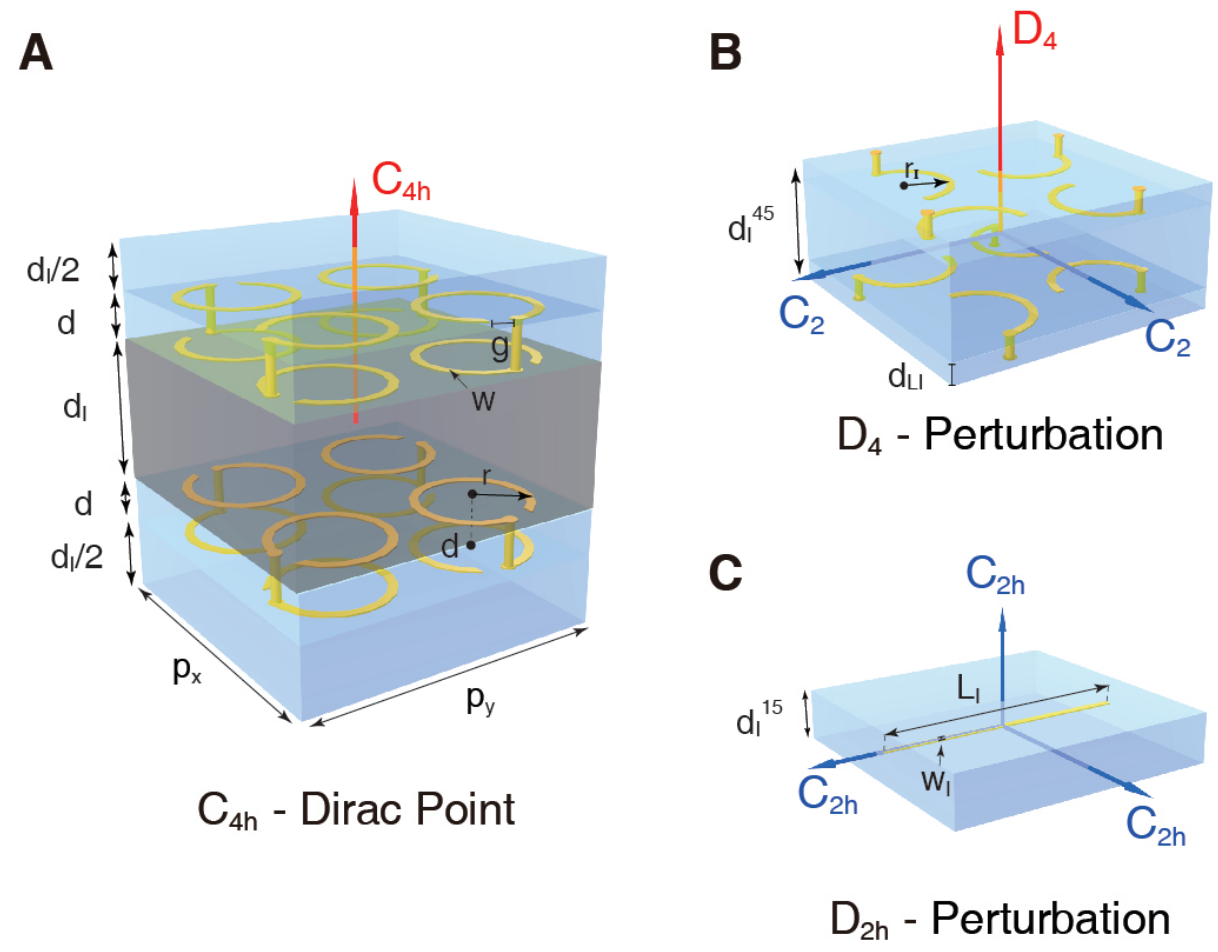

Fig. S3. Details of the designed Weyl surface metamaterials, with all the geometric parameters labeled in the plots. 

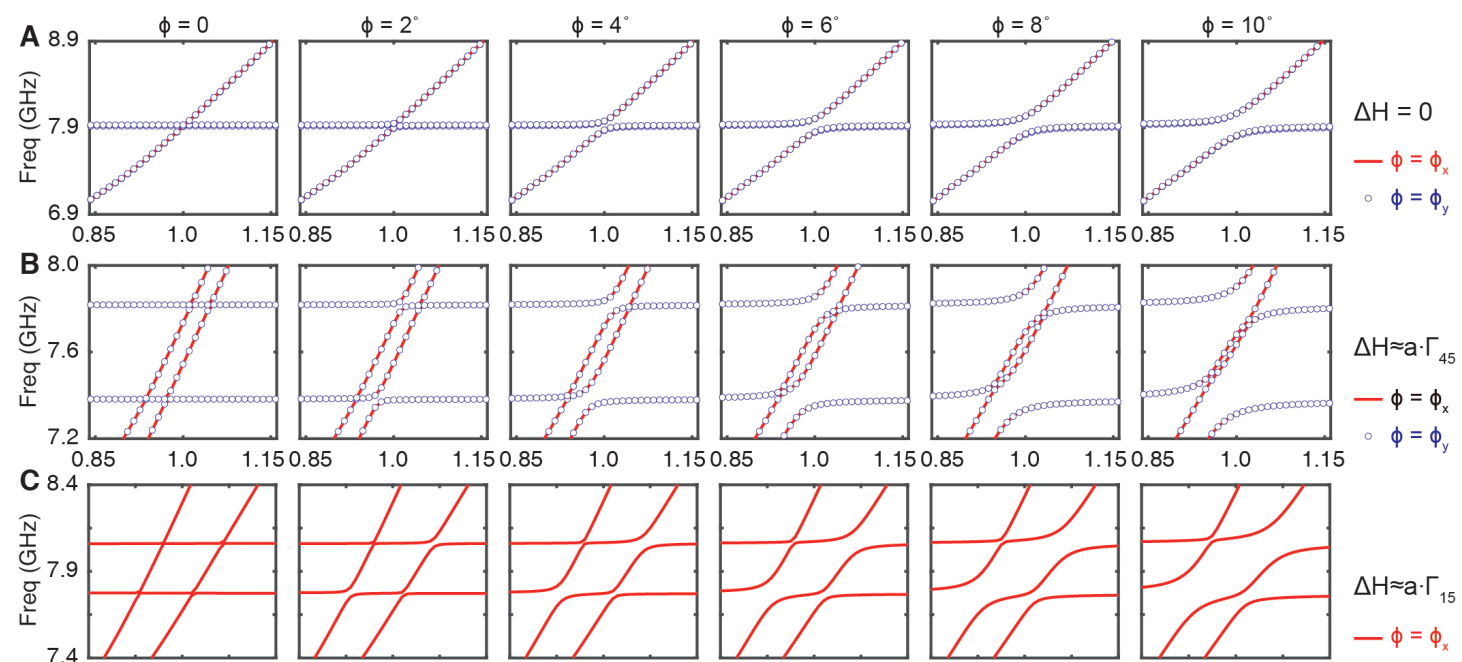

$\Delta \mathrm{H} \approx \mathrm{a} \cdot \Gamma_{15}$
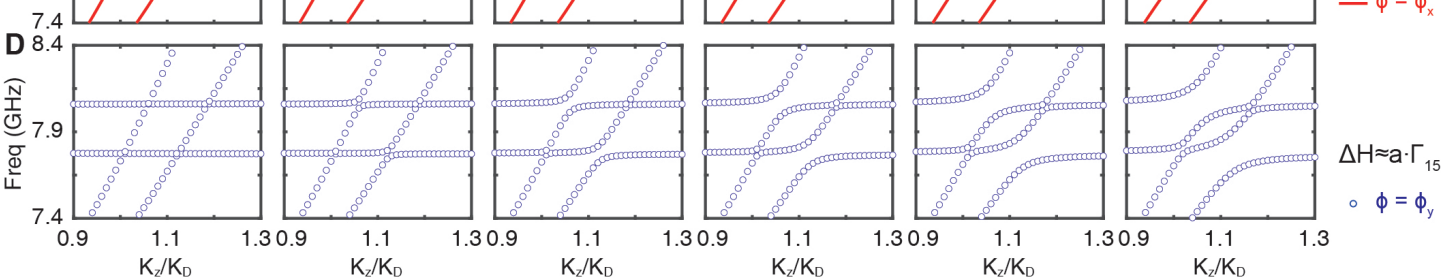

Fig. S4. Simulation results of the dispersion of the designed Weyl surface metamaterial. (A) is the dispersion of the designed 3D Dirac point metamaterial, (B-D) are the dispersion of the designed 5D linked Weyl surface metamaterials with (B) $\Delta H=a \cdot \Gamma_{45}+b \cdot \Gamma_{12}$ and (C-D) $\Delta H=a \cdot \Gamma_{15}+b \cdot \Gamma_{24}$. 

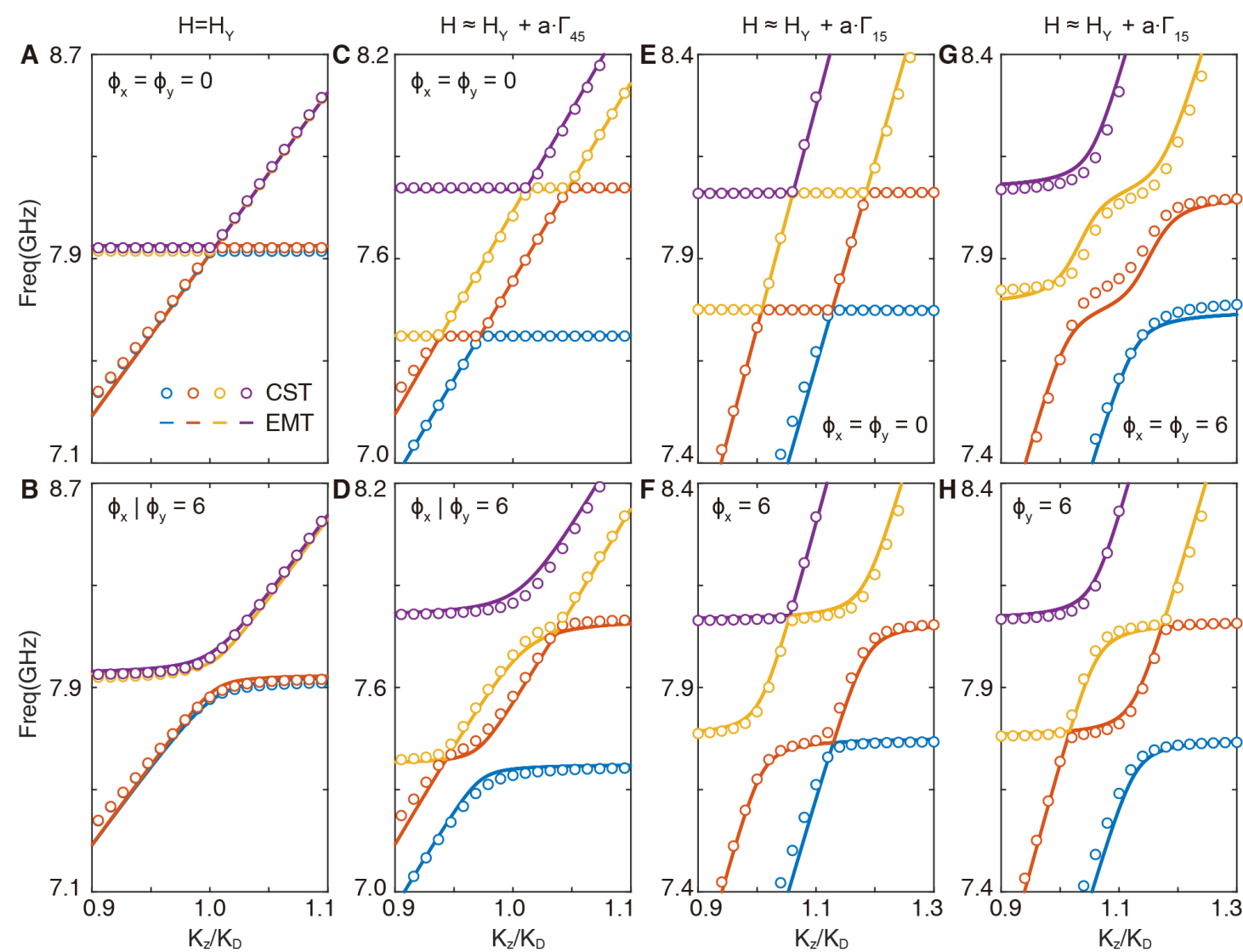

Fig. S5. Schematic diagram of retrieving the effective medium model for the designed Weyl surface metamaterials. (A-B) is the dispersion of the designed 3D Dirac point metamaterial spectrum along $k_{z}$, for various $k_{x}$ and $k_{y}$ components with $k_{i}=\Phi_{i} / P_{i}$. (C-D) is the corresponding dispersion of the designed 5D linked Weyl surface metamaterial with $\Delta H \approx a \cdot \Gamma_{45}$, while (E-H) is the corresponding of $\Delta H \approx a \cdot \Gamma_{15}$. The data are calculated by CST simulations (circles) and the fitted effective medium theory (lines). 

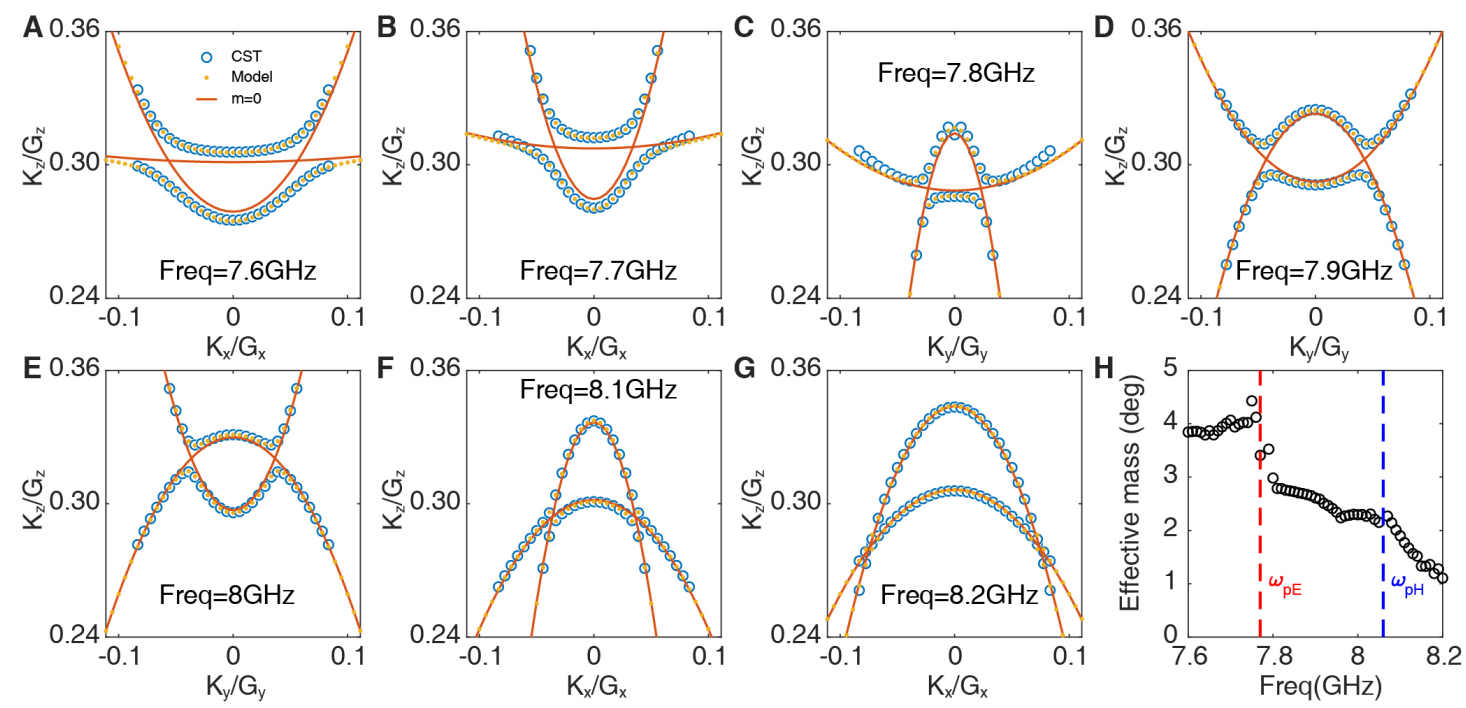

Fig. S6. Fitted EFS data for the designed linked Weyl surface metamaterial with $\Delta H \approx a \cdot \Gamma_{15}$.

(A-G) show EFS data from CST simulation (blue circles) and Fitted data with zero (red lines) and nonzero (yellow dotted lines) equivalent mass terms at different frequencies. (H) shows the frequencydependent fitted equivalent mass. 


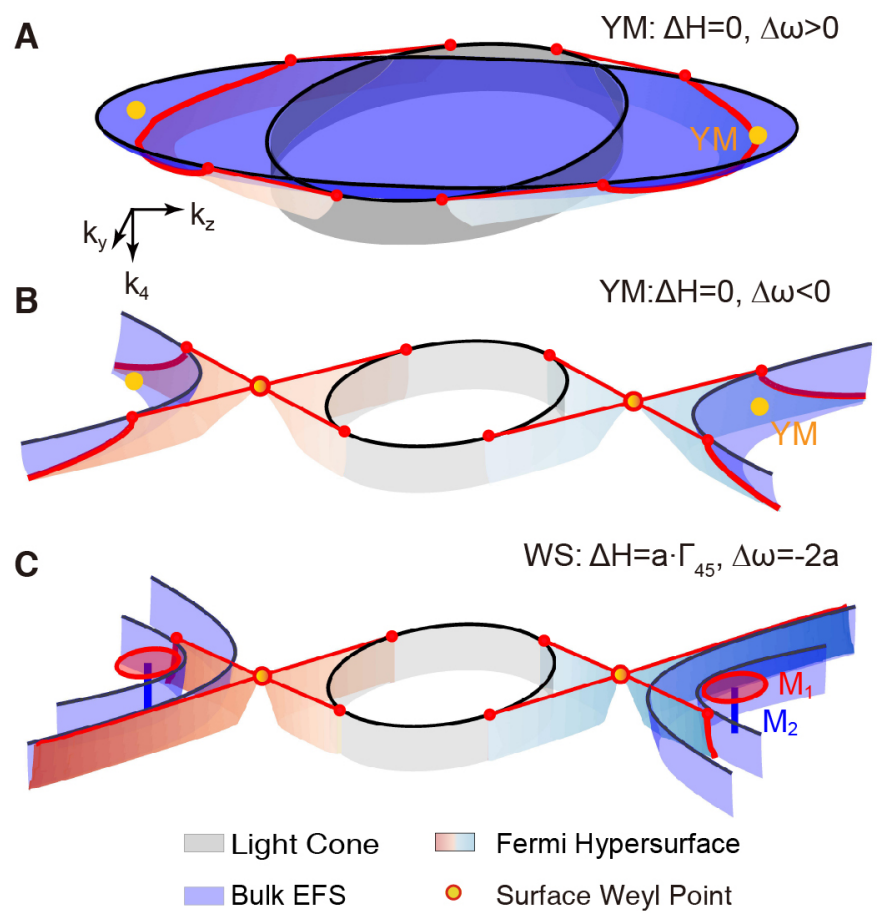

Fig. S7. The boundary states of the Yang monopole and Weyl surface metamaterial confined to the $\mathbf{x}$-direction boundary. Illustration of the light cone, bulk EFS, Fermi hypersurface and surface Weyl arc point in 5D K-space for both (A-B) Yang monopole and (C) Weyl surface with $\Delta H=a \cdot \Gamma_{45}$, with one dimension $k_{5}$ hidden. The yellows balls, red circles, and blue lines represent the Yang monopoles and the cross-sections of different Weyl surfaces, respectively. For an ideal EMT model that satisfies the ideal electromagnetic duality condition $\kappa=1$, only the boundary states for the top surface are presented. 

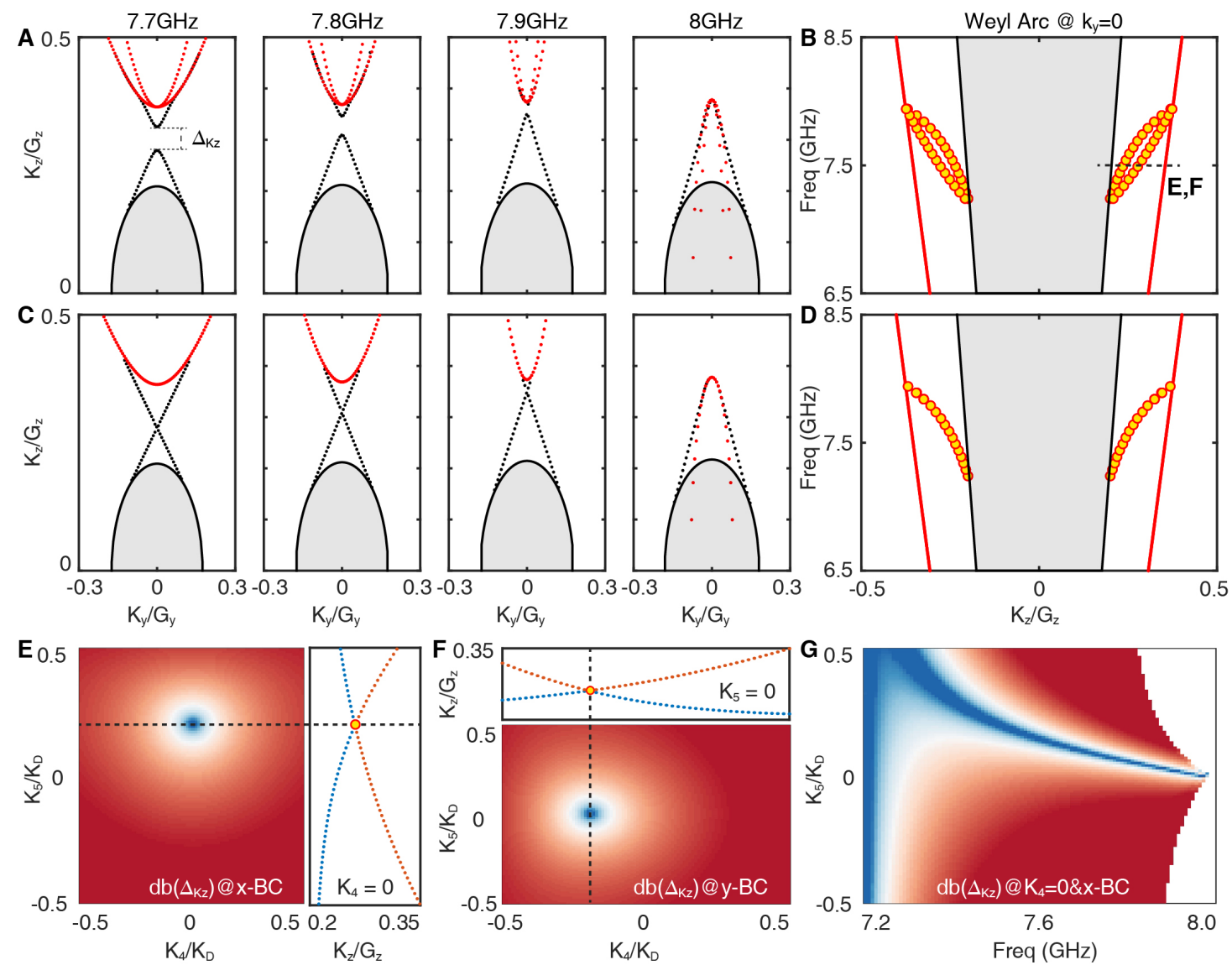

Fig. S8. Schematic of the surface state response of the Dirac metamaterial. (A) Fermi arc and (B) 1D Weyl arc distributions of the Dirac metamaterial with fitted parameters. (C-D) are the correspondences of (A-B) with perfect effective electromagnetic duality $\kappa=1$. (E-F) Numerical verifications for the existence of the surface Weyl point in full 4D synthetic space of the boundary, with boundary along (E) x-direction and (F) y-direction at a fixed frequency $7.5 \mathrm{GHz}$. (G) Numerically solution of the Weyl Arc's location in the synthetic $k_{5}$ dimension at different frequencies. 
A

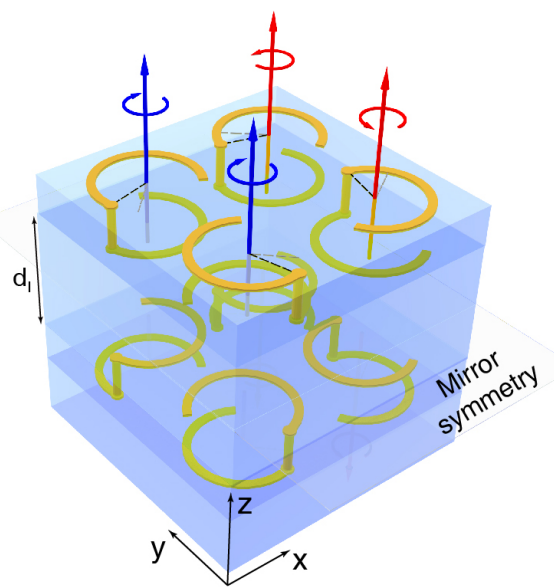

B

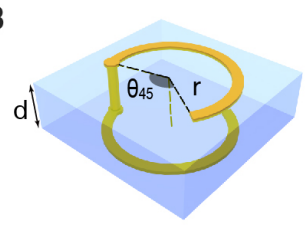

C

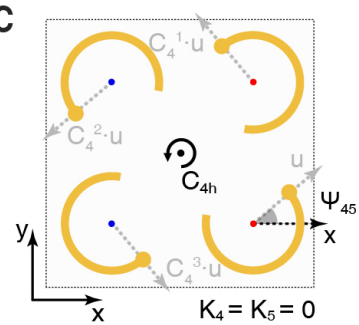

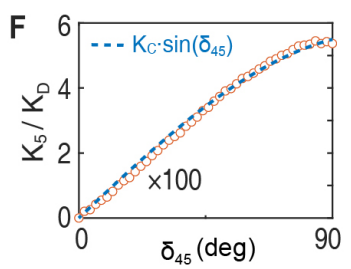

D

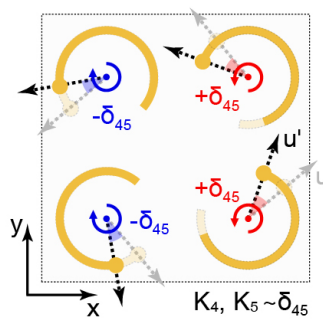

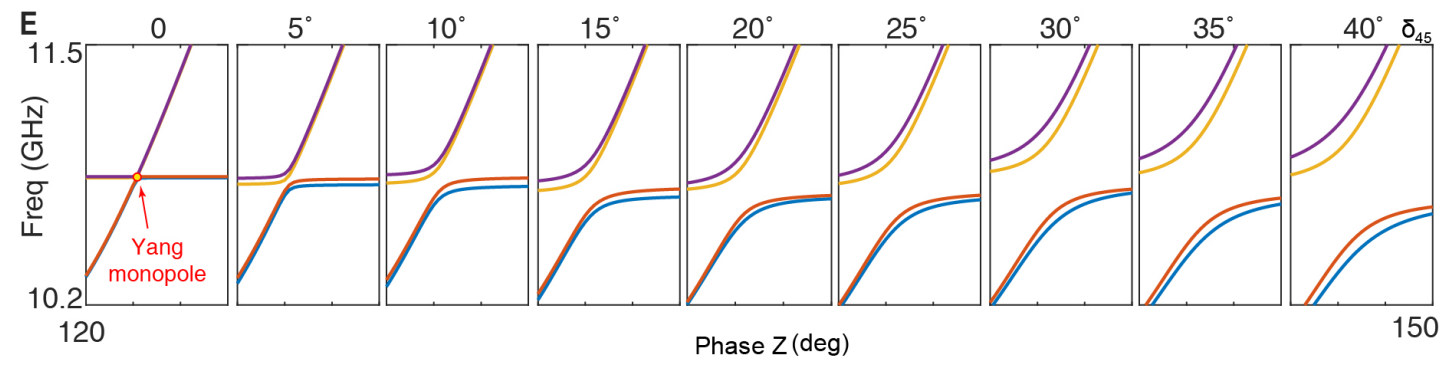

Fig. S9. Schematic diagram of the designed Yang monopole metamaterials with different synthetic momenta. (A)-(D) the configuration of a metamaterial exhibiting Yang monopole with tunable $k_{4}$ and $k_{5}$. (E) illustrates the band dispersion of the designed metamaterial along $k_{z}$, with increasing synthetic momentum $k_{5}$ tuned by the angle $\delta_{45}$. (F) shows the fitted value of $k_{5}$. The details of the structure are: $d_{I}=2.0 \mathrm{~mm}, d=1.0 \mathrm{~mm}, r=1.2 \mathrm{~mm}, \theta_{45}=2.3 \mathrm{rad}, \psi_{45}=\pi / 4$, and the other parameters are the same as those in Fig. S2A. 
A
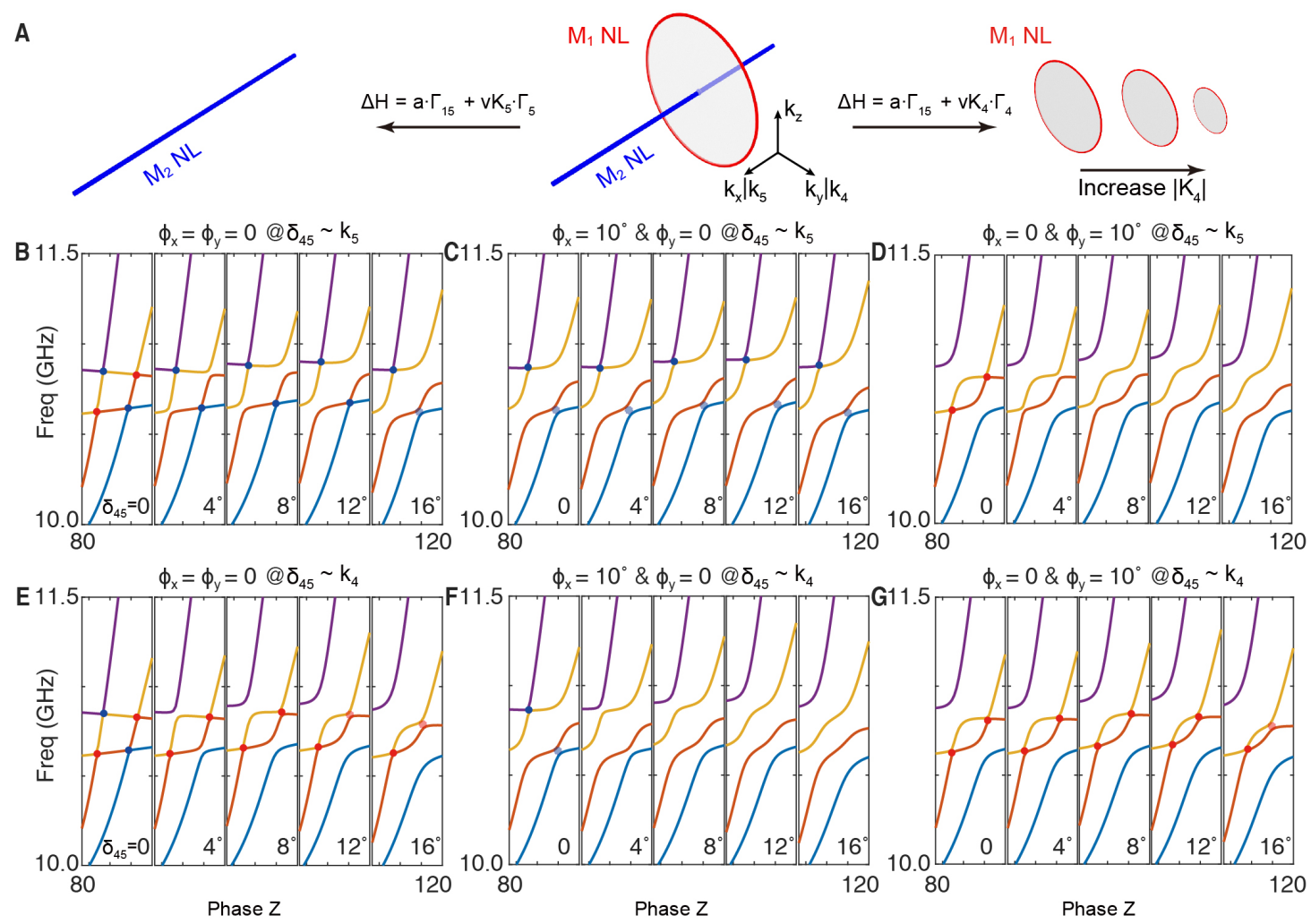

Fig. S10. The effect of the synthetic momenta on a Weyl surfaces. (A) shows the cross-section of the linked Weyl surfaces with $\Delta H=a \cdot \Gamma_{15}$ in the 3D real momentum subspace at a nonzero synthetic momentum $k_{4}$ or $k_{5}$. (B-G) shows the evolution of the band diagram along $k_{z}$ with various synthetic momenta, and for different combinations of $k_{x}$ and $k_{y}$. The red dots and blue dots denote the locations of the projected Weyl surfaces. 


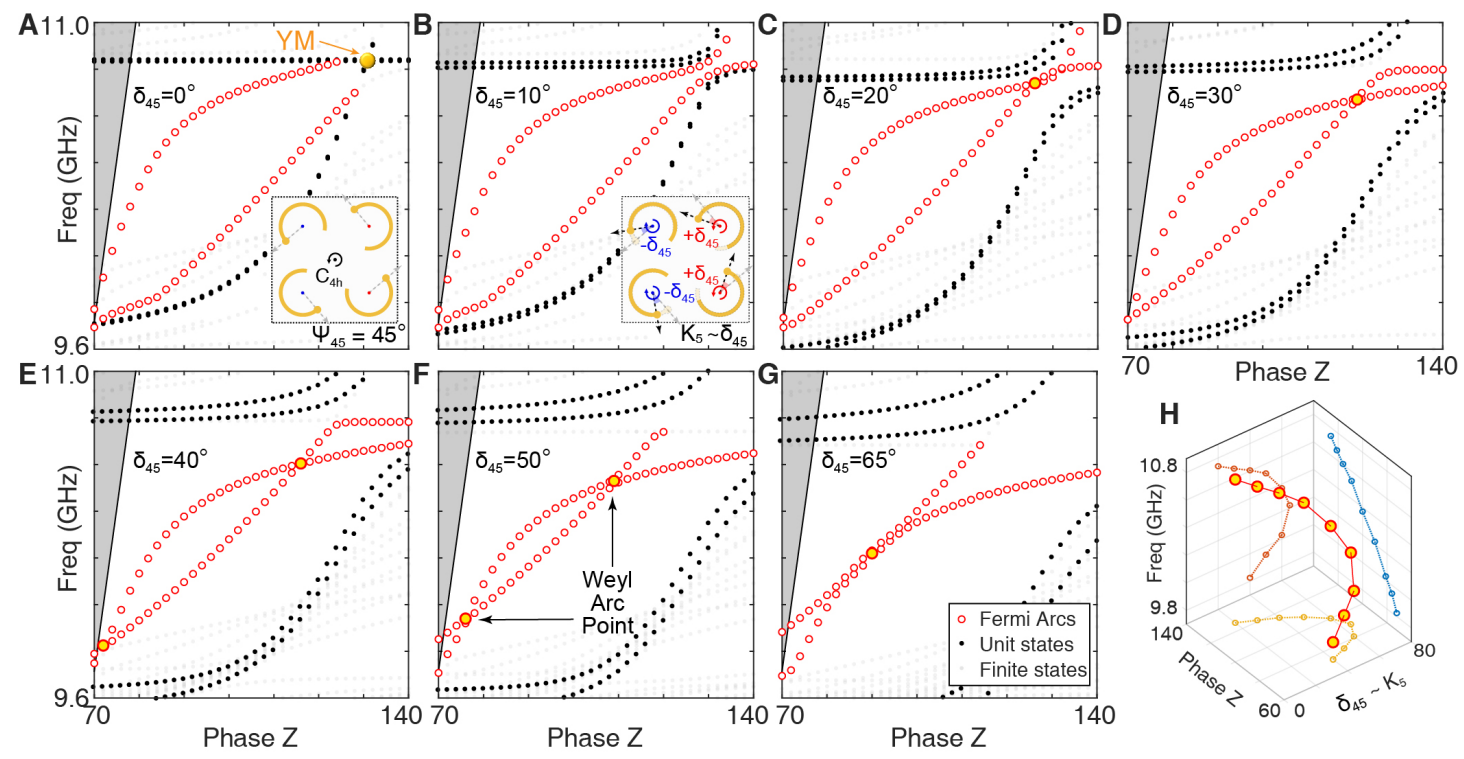

Fig. S11. The boundary states of Yang monopole metamaterials at different $\boldsymbol{k}_{5}$. (A-G) The simulated bulk/surface states of the Yang monopole system, with an increasing synthetic momentum $k_{5}$ (controlled by $\delta_{45}$ ). The orange circles represent the location of the surface Weyl points at the crossing points between two Fermi arcs. (H) The dispersion of the Weyl arc. 

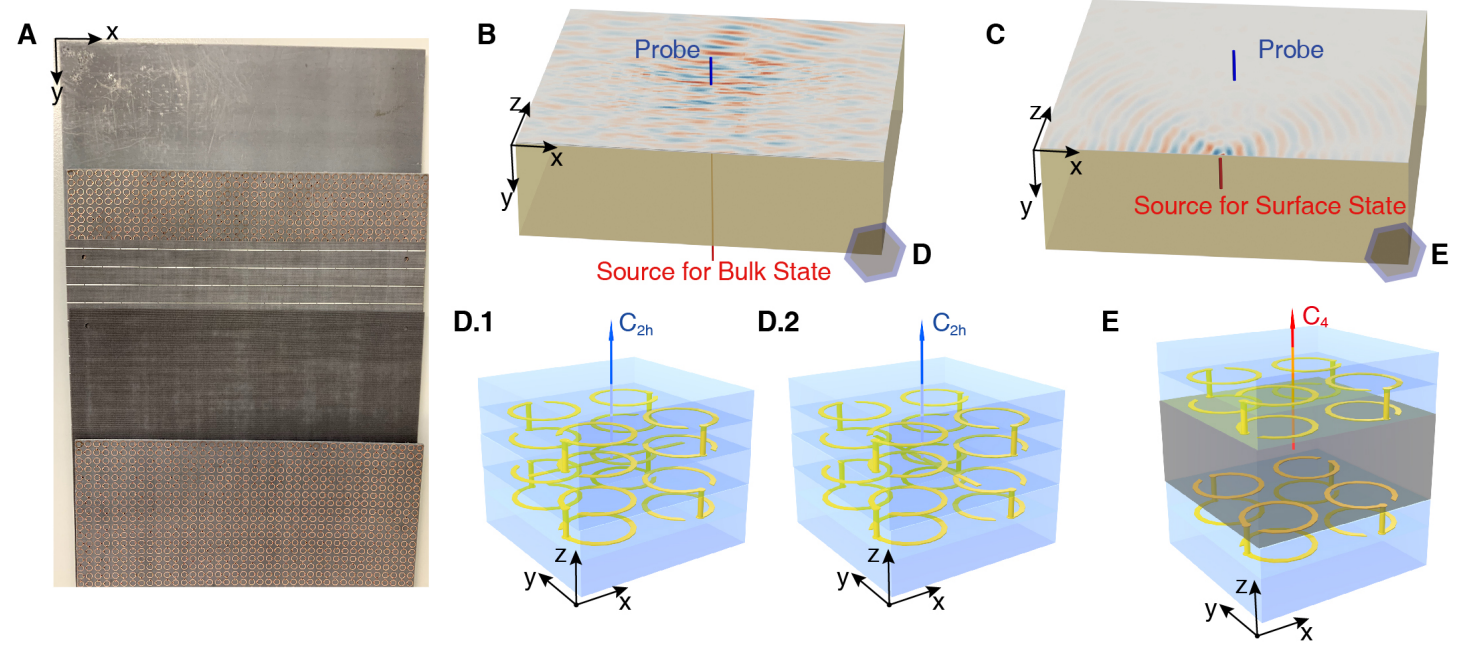

Fig. S12. Illustration of the sample and experimental setup. (A) shows the layers that are combined to form a structure with $\Delta H \approx a \cdot \Gamma_{15}$. (B-C) show the setup mainly probing (B) bulk and (C) surface state. (D-E) shows the unit cell configurations. 

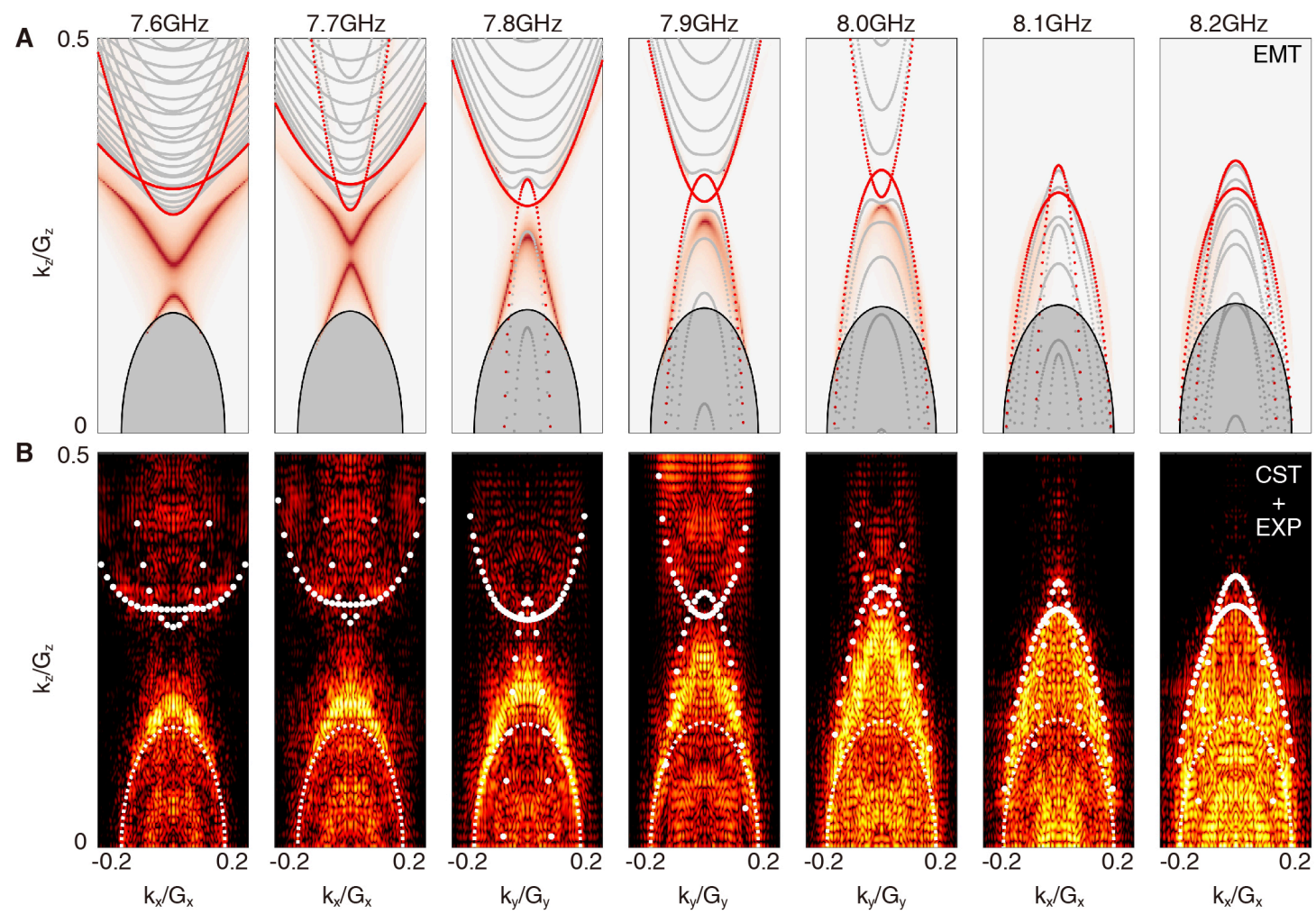

Fig. S13. The projected bulk/surface states of the Weyl surface system with $\Delta H \approx a \cdot \Gamma_{15}$. (A) shows the projected bulk states and the Fermi Arcs' location calculated by the EMT model. (B) shows the excited bulk/surface states in experimental measurements, with bulk state boundaries calculated by CST simulations. 


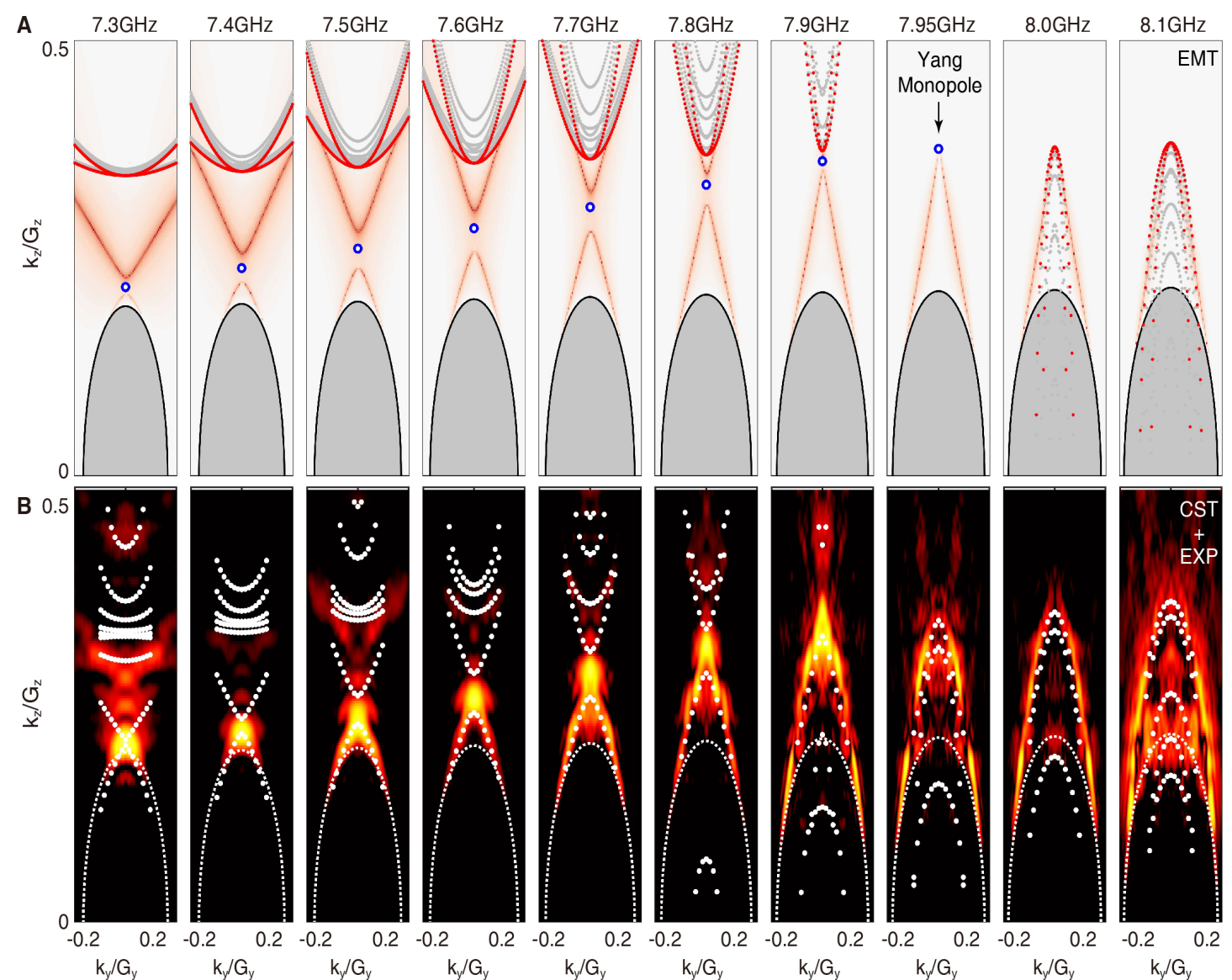

Fig. S14. The projected bulk/surface states of the Yang monopole system. (A) shows the projected bulk/surface states and the projection location of the predicted surface Weyl point (blue circles), calculated by the EMT model. (B) shows the excited bulk/surface states in experimental measurements, with numerical results (white points) calculated by CST simulations. 


\section{$\underline{\text { Reference }}$}

1. A. A. Burkov, Topological semimetals. Nat. Mater. 15, 1145-1148 (2016). doi: $10.1038 / \mathrm{nmat} 4788$

2. A. H. Castro Neto, F. Guinea, N. M. R. Peres, K. S. Novoselov, A. K. Geim, The electronic properties of graphene. Rev. Mod. Phys. 81, 109-162 (2009). doi: 10.1103/RevModPhys.81.109

3. C. L. Kane, E. J. Mele, Quantum Spin Hall Effect in Graphene. Phys. Rev. Lett. 95, 226801 (2005) doi: 10.1103/PhysRevLett.95.226801

4. X. Wan, A. M. Turner, A. Vishwanath, S. Y. Savrasov, Topological semimetal and Fermi-arc surface states in the electronic structure of pyrochlore iridates. Phys. Rev. B 83, 205101 (2011) doi: 10.1103/PhysRevB.83.205101

5. S.-Y. Xu, I. Belopolski, N. Alidoust, M. Neupane, G. Bian, C. Zhang, R. Sankar, G. Chang, Z. Yuan, C.-C. Lee, S.-M. Huang, H. Zheng, J. Ma, D. S. Sanchez, B. Wang, A. Bansil, F. Chou, P. P. Shibayev, H. Lin, S. Jia, M. Z. Hasan, Discovery of a Weyl fermion semimetal and topological Fermi arcs. Science 349, 613-617 (2015). doi: 10.1126/science.aaa9297

6. L. Lu, Z. Wang, D. Ye, L. Ran, L. Fu, J. D. Joannopoulos, M. Soljačić, Experimental observation of Weyl points. Science 349, 622-624 (2015). doi: 10.1126/science.aaa9273

7. B. Q. Lv, H. M. Weng, B. B. Fu, X. P. Wang, H. Miao, J. Ma, P. Richard, X. C. Huang, L. X. Zhao, G. F. Chen, Z. Fang, X. Dai, T. Qian, H. Ding, Experimental Discovery of Weyl Semimetal TaAs. Phys. Rev. X 5, 031013 (2015). doi: 10.1103/PhysRevX.5.031013

8. B. Yang, Q. Guo, B. Tremain, R. Liu, L. E. Barr, Q. Yan, W. Gao, H. Liu, Y. Xiang, J. Chen, C. Fang, A. Hibbins, L. Lu, S. Zhang, Ideal Weyl points and helicoid surface states in artificial photonic crystal structures. Science 359, 1013-1016 (2018). doi: 10.1126/science.aaq1221

9. N. P. Armitage, E. J. Mele, A. Vishwanath, Weyl and Dirac semimetals in three-dimensional solids. Rev. Mod. Phys. 90, 015001 (2018). doi: 10.1103/RevModPhys.90.015001

10. T. Ozawa, H. M. Price, A. Amo, N. Goldman, M. Hafezi, L. Lu, M. C. Rechtsman, D. Schuster, J. Simon, O. Zilberberg, I. Carusotto, Topological photonics. Rev. Mod. Phys. 91, 015006 (2019). doi: 10.1103/RevModPhys.91.015006

11. M. Kim, Z. Jacob, J. Rho, Recent advances in 2D, 3D and higher-order topological photonics. Light Sci. Appl. 9, 130 (2020). doi: 10.1038/s41377-020-0331-y

12. M. I. Shalaev, W. Walasik, A. Tsukernik, Y. Xu, N. M. Litchinitser, Robust topologically protected transport in photonic crystals at telecommunication wavelengths. Nat. Nanotechnol. 14, 31-34 (2019). doi: 10.1038/s41565-018-0297-6

13. N. E. Bonesteel, L. Hormozi, G. Zikos, S. H. Simon, Braid topologies for quantum computation. Phys. Rev. Lett. 95, 140503 (2005). doi: 10.1103/PhysRevLett.95.140503

14. S. C. Zhang, J. Hu, A four-dimensional generalization of the quantum Hall effect. Science 294, 823-828 (2001). doi: 10.1126/science.294.5543.823

15. X. L. Qi, T. L. Hughes, S. C. Zhang, Topological field theory of time-reversal invariant insulators. Phys. Rev. B 78, 195424 (2008). doi: 10.1103/PhysRevB.78.195424

16. H. M. Price, O. Zilberberg, T. Ozawa, I. Carusotto, N. Goldman, Four-Dimensional Quantum Hall Effect with Ultracold Atoms. Phys. Rev. Lett. 115, 195303 (2015). doi: 10.1103/PhysRevLett.115.195303

17. M. Lohse, C. Schweizer, H. M. Price, O. Zilberberg, I. Bloch, Exploring 4D quantum Hall physics with a 2D topological charge pump. Nature 553, 55-58 (2018). doi: $\underline{10.1038 / \text { nature25000 }}$ 
18. R. Yu, Y. X. Zhao, A. P. Schnyder, 4D spinless topological insulator in a periodic electric circuit. Natl. Sci.Rev. 7, 1288-1295 (2020). doi: 10.1093/nsr/nwaa065

19. O. Zilberberg, S. Huang, J. Guglielmon, M. Wang, K. P. Chen, Y. E. Kraus, M. C. Rechtsman, Photonic topological boundary pumping as a probe of 4D quantum Hall physics. Nature 553, 59-62 (2018). doi: 10.1038/nature25011

20. C. N. Yang, Generalization of Dirac's monopole to SU2 gauge fields. J. Phys. A Math. Theor. 19, 320-328 (1978). doi: 10.1063/1.523506

21. S. Sugawa, F. Salces-Carcoba, A. R. Perry, Y. Yue, I. B. Spielman, Second Chern number of a quantum-simulated non-Abelian Yang monopole. Science 360, 1429-1434 (2018). doi: $\underline{10.1126 / \text { science.aam } 9031}$

22. K. Hasebe, SO(5) Landau models and nested Nambu matrix geometry. Nucl. Phys. B 956, 115012 (2020). doi: 10.1016/j.nuclphysb.2020.115012

23. B. Lian, S. C. Zhang, Five-dimensional generalization of the topological Weyl semimetal. Phys. Rev. B 94, 041105 (2016). doi: 0.1103/PhysRevB.94.041105

24. B. Lian, S. C. Zhang, Weyl semimetal and topological phase transition in five dimensions. Phys. Rev. B 95, 235106 (2017). doi: 10.1103/PhysRevB.95.235106

25. J. Y. Chen, B. Lian, S. C. Zhang, Doubling theorem and boundary states of five-dimensional Weyl semimetal. Phys. Rev. B 100, 075112 (2019). doi: 10.1103/PhysRevB.100.075112

26. K. Hashimoto, X. Wu, T. Kimura, Edge states at an intersection of edges of a topological material. Phys. Rev. B 95, 165443 (2017). doi: 10.1103/PhysRevB.95.165443

27. K. Hashimoto, Y. Matsuo, Universal higher-order topology from a five-dimensional Weyl semimetal: Edge topology, edge Hamiltonian, and a nested Wilson loop. Phys. Rev. B 01, 245138 (2020). doi: 10.1103/PhysRevB.101.245138

28. Q. Guo, B. Yang, L. Xia, W. Gao, H. Liu, J. Chen, Y. Xiang, S. Zhang, Three Dimensional Photonic Dirac Points in Metamaterials. Phys. Rev. Lett. 119, 213901 (2017). doi: 10.1103/PhysRevLett.119.213901

29. Q. Guo, O. You, B. Yang, J. B. Sellman, E. Blythe, H. Liu, Y. Xiang, J. Li, D. Fan, J. Chen, C. T. Chan, S. Zhang, Observation of Three-Dimensional Photonic Dirac Points and SpinPolarized Surface Arcs. Phys. Rev. Lett. 122, 203903 (2019). doi: $\underline{10.1103 / \text { PhysRevLett.122.203903 }}$

30. Materials and methods are available as supplementary materials. 